\title{
ON THE METHOD OF APPROXIMATION FOR EVOLUTIONARY INCLUSIONS OF PSEUDOMONOTONE TYPE
}

\author{
PAVLO KASYANOV, VALERY MELNIK and JOSÉ VALERO
}

(Received 28 May 2007)

\begin{abstract}
For a large class of operator inclusions, including those generated by maps of pseudomonotone type, we obtain a general theorem on existence of solutions. We apply this result to some particular examples. This theorem is proved using the method of difference approximations.
\end{abstract}

2000 Mathematics subject classification: 35K55, 58C06, 34A40, 34A60.

Keywords and phrases: differential inclusions, variational inequalities, pseudomonotone maps.

\section{Introduction}

The aim of this paper is to study the existence of solutions of abstract differential inclusions with subdifferential map $\partial \varphi$ of the type

$$
\Lambda u+\mathcal{A}(u)+\partial \varphi(u) \ni f,
$$

for a large class of operators $\mathcal{A}$ including, as a particular case, pseudomonotone operators.

When studying nonlinear evolutionary equations some standard methods have been used in the past: Faedo-Galerkin approximations, singular perturbations, difference approximations, nonlinear semigroups of operators and others (see $[2,10])$. The application of these methods to evolutionary inclusions and variational inequalities has some technical difficulties. The method of nonlinear semigroups of operators in Banach spaces was developed for evolutionary inclusions in the works of Tolstonogov [20], Tolstonogov and Umanskij [21], Barbu [2] and others. The method of singular perturbations (see Brezis [3] and Dubinski [5]) has been applied to evolutionary inclusions by Vakulenko and Melnik [23-25], whereas the method of Faedo-Galerkin approximations has been applied by Kasyanov [7-9].

This work was partially supported by the MCYT (Ministerio de Ciencia y Tecnología, Spain) and FEDER (Fondo Europeo de Desarrollo Regional) grants HA2005-0082, MTM2005-01412, MTM2005-03868, and by the Consejería de Cultura y Educación (Comunidad Autónoma de Murcia) grant 00684/PI/04.

(C) 2008 Australian Mathematical Society 0004-9727/08 \$A2.00+0.00 
In this work we apply the method of difference approximations, which is a wellknown method for differential equations [10], in order to obtain the existence of solutions of evolutionary inclusions and variational inequalities, extending in this way the results known so far to a larger class of operators.

The application of the main results to some particular differential inclusions is given in the final part of the paper.

This paper is organized as follows. In the second section we explain briefly the setting of the problem considered in this work. In the third section we define (in a general multi-valued setting) the class of $\lambda$-pseudomonotone operators, which is a generalization of the class of pseudomonotone maps, and study its properties. The main advantage of this class of maps with respect to the previous one is that the sum of two $\lambda$-pseudomonotone operators with bounded values is again a $\lambda$-pseudomonotone operator. In the fourth section we state and prove the existence of solutions for the inclusion (1). Finally, in the fifth section we apply this result to a variational inequality and also to a problem arising in control theory.

\section{Setting of the problem}

Let $\Phi$ be a separable locally convex linear topological space, and let $\Phi^{\prime}$ be the topological conjugate to $\Phi$. By $(f, \xi)$ we denote the canonical pairing of $f \in \Phi^{\prime}$ and $\xi \in \Phi$.

Let the spaces $\mathcal{V}, \mathcal{H}$ and $\mathcal{V}^{\prime}$ be given. Moreover,

$$
\Phi \subset \mathcal{V} \subset \Phi^{\prime}, \quad \Phi \subset \mathcal{H} \subset \Phi^{\prime}, \quad \Phi \subset \mathcal{V}^{\prime} \subset \Phi^{\prime},
$$

with continuous and dense embeddings. We assume that $\mathcal{H}$ is a Hilbert space with the scalar product $\left(h_{1}, h_{2}\right) \mathcal{H}$ and norm $\|h\|_{\mathcal{H}}, \mathcal{V}$ is a reflexive separable Banach space with norm $\|v\|_{\mathcal{V}}$ and $\mathcal{V}^{\prime}$ is the conjugate to $\mathcal{V}$ with the norm $\|f\|_{\mathcal{V}^{\prime}}$ associated with the bilinear form $(\cdot, \cdot)_{\mathcal{H}}$.

If $\xi, \psi \in \Phi$, then $(\xi, \psi)=(\xi, \psi)_{\mathcal{H}}$, that is it coincides with the scalar product in $\mathcal{H}$.

Let $\mathcal{V}=\mathcal{V}_{1} \cap \mathcal{V}_{2}$ and $\|\cdot\| \mathcal{V}=\|\cdot\|_{\mathcal{V}_{1}^{\prime}}+\|\cdot\|_{\mathcal{V}_{2}^{\prime}}$, where $\left(\mathcal{V}_{i},\|\cdot\| \mathcal{V}_{i}\right), i=\overline{1,2}$, are reflexive separable Banach spaces and the embeddings $\Phi \subset \mathcal{V}_{i} \subset \Phi^{\prime}$ and $\Phi \subset \mathcal{V}_{i}^{\prime} \subset \Phi^{\prime}$ are dense and continuous. The spaces $\left(\mathcal{V}_{i}^{\prime},\|\cdot\|_{\mathcal{V}_{i}^{\prime}}\right), i=\overline{1,2}$, are the topologically conjugates to $\left(\mathcal{V}_{i},\|\cdot\| \mathcal{V}_{i}\right)$. Then $\mathcal{V}^{\prime}=\mathcal{V}_{1}^{\prime}+\mathcal{V}_{2}^{\prime}$.

Let $A: \mathcal{V}_{1} \rightrightarrows \mathcal{V}_{1}^{\prime}$ be a multi-valued map with non-empty convex closed bounded values. Let $\varphi: \mathcal{V}_{2} \rightarrow \mathbb{R}$ be a convex lower semicontinuous functional, and let $\Lambda: \mathcal{V} \rightarrow$ $\mathcal{V}^{\prime}$ be an unbounded operator with domain $D\left(\Lambda ; \mathcal{V}, \mathcal{V}^{\prime}\right)$. We consider the following problem:

$$
\begin{gathered}
u \in D\left(\Lambda ; \mathcal{V}, \mathcal{V}^{\prime}\right), \\
\Lambda u+\mathcal{A}(u)+\partial \varphi(u) \ni f,
\end{gathered}
$$

where $f \in \mathcal{V}^{\prime}$ is a fixed element, and $\partial \varphi: \mathcal{V}_{2} \rightrightarrows \mathcal{V}_{2}^{\prime}$ is the subdifferential of the functional $\varphi$ (see [18]). 
Our aim is to prove the existence of solutions by the method of finite differences (see [10, Chapter 2.7]).

\section{Preliminary results}

Let us assume that the space $\Phi$ is dense in $\left(\mathcal{V} \cap \mathcal{V}^{\prime},\|v\|_{\mathcal{V}}+\|v\|_{\mathcal{V}^{\prime}}\right)$. It follows from this assumption that

$$
\mathcal{V} \cap \mathcal{V}^{\prime} \subset \mathcal{H}
$$

In fact, if $\xi \in \Phi$, then $\|v\|_{\mathcal{H}}^{2} \leq\|v\|_{\mathcal{V}^{\prime}}\|v\|_{\mathcal{V}}$, so that (5) follows.

REMARK 1. If $\mathcal{V} \subset \mathcal{H}$ with dense and continuous embedding, it is possible to avoid the space $\Phi$. Identifying $\mathcal{H}$ and $\mathcal{H}^{\prime}$ we obtain the following embeddings:

$$
\mathcal{V} \subset \mathcal{H} \subset \mathcal{V}^{\prime}
$$

Definition 2. The family of maps $\{G(s)\}_{s \geq 0}$ is said to be a semigroup of class $C_{0}$ in a Banach space $X$ if $G(s) \in \mathcal{L}(X ; X)$, for any $s \geq 0, G(0)=\mathrm{Id}, G(s+t)=$ $G(s) \circ G(t)$, for all $s, t \geq 0$, and $G(t) x \rightarrow x$ as $t \rightarrow 0^{+}$, for all $x \in X$.

Let the family of maps $\{G(s)\}_{s \geq 0}$ be a semigroup of class $C_{0}$ on $\mathcal{V}, \mathcal{H}, \mathcal{V}^{\prime}$, that is, there are three semigroups, defined in the spaces $\mathcal{V}, \mathcal{H}$, and $\mathcal{V}^{\prime}$, respectively, which coincide on $\Phi$. Each of them will be denoted by $\{G(s)\}_{s \geq 0}$. Moreover, we assume the following:

$$
\begin{gathered}
\{G(s)\}_{s \geq 0} \text { is a non-expansive semigroup on } \mathcal{H}, \\
\text { that is }\|G(s)\|_{\mathcal{L}(\mathcal{H} ; \mathcal{H}) \leq 1} \text { for all } s \geq 0 .
\end{gathered}
$$

Furthermore, let $-\Lambda$ be the infinitesimal generator of the semigroup $\{G(s)\}_{s \geq 0}$ with domain $D(\Lambda ; \mathcal{V})$ (respectively $D(\Lambda ; \mathcal{H})$ or $D\left(\Lambda ; \mathcal{V}^{\prime}\right)$ ) in $\mathcal{V}$ (respectively $\mathcal{H}$ or $\mathcal{V}^{\prime}$ ). It is well known [18] that such a generator exists. Moreover, it is a densely defined closed linear operator in the space $\mathcal{V}$ (respectively in $\mathcal{H}$ or $\mathcal{V}^{\prime}$ ).

Let $\left\{G^{*}(s)\right\}_{s \geq 0}$ be the semigroup conjugated to $G(s)$. Let $-\Lambda^{*}$ be the infinitesimal generator of the semigroup $\left\{G^{*}(s)\right\}_{s \geq 0}$ with domain $D\left(\Lambda^{*} ; \mathcal{V}\right)$ in $\mathcal{V}, D\left(\Lambda^{*} ; \mathcal{H}\right)$ in $\mathcal{H}$ and $D\left(\Lambda^{*} ; \mathcal{V}^{\prime}\right)$ in $\mathcal{V}^{\prime}$. The operator $\Lambda^{*}$ in $\mathcal{H}$ (respectively in $\mathcal{V}$ or $\mathcal{V}^{\prime}$ ) is conjugated to the operator $\Lambda$ in $\mathcal{H}$ (respectively in $\mathcal{V}$ or $\mathcal{V}^{\prime}$ ).

Lemma 3. The sets $D\left(\Lambda ; \mathcal{V}^{\prime}\right) \cap \mathcal{V}$ and $D\left(\Lambda^{*} ; \mathcal{V}^{\prime}\right) \cap \mathcal{V}$ are dense in $\mathcal{V}$.

PROOF. In fact, for any $u \in \mathcal{V}$ and $\varepsilon>0$ there exists $\xi \in \Phi$ such that $\|u-\xi\| \mathcal{V}<\varepsilon$. Then $\xi_{n}:=(I-1 / n \Lambda)^{-1} \xi \in D\left(\Lambda ; \mathcal{V}^{\prime}\right) \cap \mathcal{V}, \xi_{n} \rightarrow \xi$ in $\mathcal{V}$ as $n \rightarrow \infty$

Now we shall define $\Lambda$ as an unbounded operator, which operates from $\mathcal{V}$ in $\mathcal{V}^{\prime}$ with domain $D\left(\Lambda ; \mathcal{V}, \mathcal{V}^{\prime}\right)$. Let us put

$D\left(\Lambda ; \mathcal{V}, \mathcal{V}^{\prime}\right)=\left\{v \in \mathcal{V} \mid\right.$ the form $w \rightarrow\left\langle v, \Lambda^{*} w\right\rangle_{\mathcal{V}}$ is continuous in $D\left(\Lambda^{*} ; \mathcal{V}^{\prime}\right) \cap \mathcal{V}$

with respect to the topology induced by the space $\mathcal{V}$ \}. 
Then there is a unique element $\xi_{v} \in \mathcal{V}^{\prime}$ such that $\left\langle v, \Lambda^{*} w\right\rangle_{\mathcal{V}}=\left\langle\xi_{v}, w\right\rangle_{\mathcal{V}}$. If $v \in D\left(\Lambda ; \mathcal{V}^{\prime}\right) \cap \mathcal{V}$, then $\xi_{v}=\Lambda v$. Thus, generally speaking, we can put $\xi_{v}=\Lambda v$, and hence

$$
\left\langle v, \Lambda^{*} w\right\rangle_{\mathcal{V}}=\langle\Lambda v, w\rangle_{\mathcal{V}} \quad \text { for all } w \in D\left(\Lambda^{*} ; \mathcal{V}^{\prime}\right) \cap \mathcal{V}
$$

Defining on $D\left(\Lambda ; \mathcal{V}, \mathcal{V}^{\prime}\right)$ the norm $\|v\|_{\mathcal{V}}+\|\Lambda v\|_{\mathcal{V}^{\prime}}$, we obtain a Banach space. We define in a similar way the space $D\left(\Lambda^{*} ; \mathcal{V}, \mathcal{V}^{\prime}\right)$.

REMARK 4. If $\mathcal{V} \subset \mathcal{H}$ with dense and continuous embedding, then

$$
D\left(\Lambda ; \mathcal{V}, \mathcal{V}^{\prime}\right)=\mathcal{V} \cap D\left(\Lambda ; \mathcal{V}^{\prime}\right) \quad \text { and } \quad D\left(\Lambda^{*} ; \mathcal{V}, \mathcal{V}^{\prime}\right)=\mathcal{V} \cap D\left(\Lambda^{*} ; \mathcal{V}^{\prime}\right)
$$

In the case when $\mathcal{V}$ is not contained in $\mathcal{H}$, we shall assume that

$$
\begin{gathered}
\mathcal{V} \cap D\left(\Lambda ; \mathcal{V}^{\prime}\right) \text { is dense in } D\left(\Lambda ; \mathcal{V}, \mathcal{V}^{\prime}\right) \\
\mathcal{V} \cap D\left(\Lambda^{*} ; \mathcal{V}^{\prime}\right) \text { is dense in } D\left(\Lambda^{*} ; \mathcal{V}, \mathcal{V}^{\prime}\right)
\end{gathered}
$$

REMARK 5. It is known [10, Chapter 2] that

$\langle\Lambda v, v\rangle_{\mathcal{V}} \geq 0 \quad$ for all $v \in D\left(\Lambda ; \mathcal{V}, \mathcal{V}^{\prime}\right),\left\langle\Lambda^{*} v, v\right\rangle_{\mathcal{V}} \geq 0 \quad$ for all $v \in D\left(\Lambda^{*} ; \mathcal{V}, \mathcal{V}^{\prime}\right)$

Let us introduce some new notation. Let $X$ be some Banach space. For a non-empty subset $B \subset X$ we shall denote the closed convex hull of $B$ by $\overline{c o}(B):=$ $\operatorname{cl}_{Y}(\operatorname{co}(B))$. For the multi-valued map $A: X \rightrightarrows X^{\prime}$ we define the upper $[A(y), \omega]_{+}=$ $\sup _{d \in A(y)}\langle d, w\rangle_{X}$ and lower $[A(y), \omega]_{-}=\inf _{d \in A(y)}\langle d, w\rangle_{X}$ support functions, where $y, \omega \in X$, and also the upper $\|A(y)\|_{+}=\sup _{d \in \mathcal{A}(y)}\|d\|_{X^{\prime}}$ and lower $\|A(y)\|_{-}=$ $\inf _{d \in \mathcal{A}(y)}\|d\|_{X^{\prime}}$ norms. The properties of the given maps are considered in the works $[11,12,15]$. Later on $y_{n} \rightarrow y$ in $X$ means that $y_{n}$ converges weakly to $y$ in the space $X$. If the space $X$ is reflexive, then $y_{n} \rightarrow y$ in $X^{\prime}$ will mean that $y_{n}$ converges weakly to $y$ in the space $X^{\prime}$. If not, then $y_{n}$ converges to $y$ weakly star in the space $X^{\prime}$.

Denote by $C_{v}\left(X^{\prime}\right)\left(\mathcal{B}\left(X^{\prime}\right)\right)$ the set of all non-empty, convex, weakly star closed subsets of $X^{\prime}$ (the set of all non-empty bounded subsets of $X^{\prime}$ ), and by $\overline{\mathrm{co}}^{*} A$ the weakly star closure of the convex hull of the set $A$ in the space $X^{\prime}$.

Proposition 6. Let $A, B: X \rightrightarrows X^{\prime}$. Then for all $y, v, v_{1}, v_{2} \in X$ the following results are true.

(1) Let $a(\cdot, \cdot): D \times X \subset X \times X \rightarrow \overline{\mathbb{R}}=\mathbb{R} \cup\{+\infty\}$. For each $y \in D \subset X$ a functional $X \ni w \mapsto a(y, w)$ is positive homogeneous convex and lower semicontinuous if and only if when there exists a multi-valued map $A: X \rightarrow 2^{X^{\prime}}$ such that $D(A)=D$ and

$$
a(y, w)=[A(y), w]_{+} \quad \text { for all } y \in D(A), w \in X .
$$

(2) $\left[A(y), v_{1}+v_{2}\right]_{+} \leq\left[A(y), v_{1}\right]_{+}+\left[A(y), v_{2}\right]_{+}$, $\left[A(y), v_{1}+v_{2}\right]_{-} \geq\left[A(y), v_{1}\right]_{-}+\left[A(y), v_{2}\right]_{-}$, $\left[A(y), v_{1}+v_{2}\right]_{+} \geq\left[A(y), v_{1}\right]_{+}+\left[A(y), v_{2}\right]_{-}$, $\left[A(y), v_{1}+v_{2}\right]_{-} \leq\left[A(y), v_{1}\right]_{+}+\left[A(y), v_{2}\right]_{-}$. 
(3) $[A(y)+B(y), v]_{+}=[A(y), v]_{+}+[B(y), v]_{+}$, $[A(y)+B(y), v]_{-}=[A(y), v]_{-}+[B(y), v]_{-}$.

(4) $[A(y), v]_{+} \leq\|A(y)\|_{+}\|v\|_{X}$, $[A(y), v]_{-} \leq\|A(y)\|_{-}\|v\|_{X}$.

(5) $[A(y), v]_{+}=\left[\overline{\mathrm{co}}^{*} A(y), v\right]_{+},[A(y), v]_{-}=\left[\overline{\mathrm{co}}^{*} A(y), v\right]_{-}$.

(6) $\|A(y)-B(y)\|_{+} \geq\left|\|A(y)\|_{+}-\|B(y)\|_{-}\right|$, $\|A(y)-B(y)\|_{-} \geq\|A(y)\|_{-}-\|B(y)\|_{+}$.

(7) $d \in \overline{\mathrm{co}}^{*} A(y)$ if and only if for all $\omega \in X[A(y), \omega]_{+} \geq\langle d, w\rangle_{X}$.

(8) $d_{H}(A(y), B(y)) \geq\left|\|A(y)\|_{+}-\|B(y)\|_{+}\right|$, $d_{H}(A(y), B(y)) \geq\left|\|A(y)\|_{-}-\|B(y)\|_{-}\right|$, where $d_{H}$ is the Hausdorff metric.

(9) For each $A, B, C: X \rightrightarrows X^{\prime}$,

$$
\begin{aligned}
\operatorname{dist}(A(y)+B(y), C(y)) & \leq \operatorname{dist}(A(y), C(y))+\operatorname{dist}(B(y), 0), \\
\operatorname{dist}(C(y), A(y)+B(y)) & \leq \operatorname{dist}(C(y), A(y))+\operatorname{dist}(0, B(y)), \\
d_{H}(A(y)+B(y), C(y)) & \leq d_{H}(A(y), C(y))+d_{H}(B(y), 0),
\end{aligned}
$$

where $\operatorname{dist}(A, B)=\sup _{a \in A} \inf _{b \in B}\|a-b\|_{X^{\prime}}$.

(10) For each $A \subset X^{\prime}$ and bounded $B \in C_{v}\left(X^{\prime}\right)$,

$$
\operatorname{dist}(A, B)=\operatorname{dist}\left(\overline{\mathrm{co}}^{*} A, B\right) .
$$

REMARK 7 . Together with the forms $[\cdot, \cdot]_{+},[\cdot, \cdot]_{-}$we shall consider the forms $[[A(y), \omega]]_{+}=\sup _{d \in A(y)}|\langle d, w\rangle| \quad$ and $\quad[[A(y), \omega]]_{-}=\inf _{d \in A(y)}|\langle d, w\rangle|$, for all $y, \omega \in X$. Thus it is obvious that

$$
\begin{aligned}
& {[A(y), \omega]_{+} \leq\left|[A(y), \omega]_{+}\right| \leq[[A(y), \omega]]_{+} \leq\|A(y)\|_{+}\|\omega\|_{X},} \\
& {[A(y), \omega]_{-} \leq\left|[A(y), \omega]_{-}\right| \leq[[A(y), \omega]]_{-} \leq\|A(y)\|_{-}\|\omega\|_{X} .}
\end{aligned}
$$

REMARK 8. Other interesting properties can be found in [14].

Proof. Properties (2)-(4) and (6)-(9) are proved directly. Property (5) is well known. Let us consider property (7). Let $d \in \overline{\mathrm{co}}^{*} A(y)$. Then for any $v \in X$ from the definition of $[\cdot, \cdot]_{+}$it follows that

$$
\langle d, v\rangle_{X} \leq\left[\overline{\mathrm{co}}^{*} A(y), v\right]_{+}=[A(y), v]_{+} .
$$

Now let the inequality

$$
[A(y), v]_{+} \geq\langle d, v\rangle_{X} \text { for all } v \in X,
$$

be valid but nevertheless $d \notin \overline{\mathrm{co}}^{*} A(y)$. The set $\overline{\mathrm{co}}^{*} A(y)$ is convex and closed in the $\sigma\left(X^{\prime} ; X\right)$-topology of the space $X^{\prime}$. Therefore, from the separability theorem there exists $v_{0} \in X$ such that

$$
\left[A(y), v_{0}\right]_{+}=\left[\overline{\mathrm{co}}^{*} A(y), v_{0}\right]_{+}<\left\langle d, v_{0}\right\rangle_{X},
$$

which contradicts the assumption. 
Now let us consider property (1). Let $A: D(A) \subset X \rightarrow 2^{X^{\prime}}$. Then for each $y \in D(A)$ the functional $X \ni v \mapsto a(y, v)=[A(y), v]_{+}$is positive homogeneous. Hence, the second property in Proposition 6 implies that it is convex. The lower semicontinuity is obvious.

Now let $X \ni v \mapsto a(y, v)$ be a positive homogeneous, convex and lower semicontinuous functional for each $y \in D \subset X$. In virtue of $a(y, 0)=0$ it is the pointwise upper bound of some set of continuous linear functionals. We denote such a set by $A(y) \subset X^{\prime}$. Thus $a(y, v)=[A(y), v]_{+}$.

Finally, let us consider property (10). Let us prove first that $\operatorname{dist}(A, B)=$ $\operatorname{dist}(\operatorname{co} A, B)$. As $A \subset \operatorname{co} A$, then $\operatorname{dist}(A, B) \leq \operatorname{dist}(\operatorname{co} A, B)$. Further, for any $\xi \in \operatorname{co} A$ there exist $y_{1}, \ldots, y_{n} \in A$ and $\alpha_{1}, \ldots, \alpha_{n} \geq 0\left(\sum_{i=1}^{n} \alpha_{i}=1\right)$, such that $\xi=\sum_{i=1}^{n} \alpha_{i} y_{i}$. Then $\operatorname{dist}(\xi, B) \leq\|\xi-v\|_{X^{\prime}}$, for all $v \in B$.

The set $B$ is bounded and weakly star closed in $X^{\prime}$. Also, for any $y \in X^{\prime}$ the functional $X^{\prime} \ni v \mapsto\|y-v\|_{X^{\prime}}$ is weakly star lower semicontinuous [17]. Hence, by virtue of the generalized Weierstrass theorem (see Lemma 19), for every $y_{i} \in A$ there exists an element $v_{i} \in B$ such that $\operatorname{dist}\left(y_{i}, B\right)=\left\|y_{i}-v_{i}\right\|_{X^{\prime}}$. From here, due to the convexity of the set $B$, we have that, for $v=\sum_{i=1}^{n} \alpha_{i} v_{i}$,

$$
\operatorname{dist}(\xi, B) \leq\|\xi-v\|_{X^{\prime}} \leq \sum_{i=1}^{n} \alpha_{i} \cdot\left\|y_{i}-v_{i}\right\|_{X^{\prime}}=\sum_{i=1}^{n} \alpha_{i} \operatorname{dist}\left(y_{i}, B\right) \leq \operatorname{dist}(A, B) .
$$

As the element $\xi \in \operatorname{co} A$ is arbitrary, then $\operatorname{dist}(\operatorname{co} A, B)=\operatorname{dist}(A, B)$.

Further, let us prove that

$$
\operatorname{dist}(\operatorname{co} A, B)=\operatorname{dist}\left(\overline{\operatorname{co}}^{*} A, B\right) .
$$

For every $\xi \in \overline{\mathrm{co}}^{*} A$ there exists a sequence $y_{n} \in c o A$ converging to $\xi$ weakly star in $X^{\prime}$. Let us prove that the functional $X^{\prime} \ni y \mapsto \operatorname{dist}(A, B)$ is weakly star lower semicompact, that is for each sequence $y_{n} \rightarrow y$ weakly star in $X^{\prime}$ there exists a subsequence $\left\{y_{n^{\prime}}\right\}$ such that

$$
\underset{n^{\prime} \rightarrow \infty}{\lim } \operatorname{dist}\left(y_{n^{\prime}}, B\right) \geq \operatorname{dist}(y, B) .
$$

Indeed, let $y_{n} \rightarrow y$ weakly star in $X^{\prime}$. For each $y_{n}$ there exists $x_{n} \in B$ such that $\operatorname{dist}\left(y_{n}, B\right)=\left\|y_{n}-x_{n}\right\|_{X^{\prime}}$. The set $B$ is weakly star compact, so that the sequence $\left\{x_{n}\right\}$ contains a subsequence $\left\{x_{n^{\prime}}\right\}$ such that $x_{n^{\prime}} \rightarrow x$ weakly star in $X^{\prime}, x \in B$. Then

$$
z_{n^{\prime}}=y_{n^{\prime}}-x_{n^{\prime}} \rightarrow z=y-x \quad \text { weakly star in } X^{\prime} .
$$

This means that

$$
\underset{n^{\prime} \rightarrow \infty}{\varliminf_{i m}} \operatorname{dist}\left(y_{n^{\prime}}, B\right)=\underset{n^{\prime} \rightarrow \infty}{\lim _{n}}\left\|y_{n^{\prime}}-x_{n^{\prime}}\right\|_{X^{\prime}} \geq\|y-x\|_{X^{\prime}} \geq \operatorname{dist}(y, B) .
$$

From here we have (passing if necessary to a subsequence) that

$$
\operatorname{dist}(\xi, B) \leq \underset{n \rightarrow \infty}{\lim _{n \rightarrow \infty}} \operatorname{dist}\left(y_{n}, B\right) \leq \operatorname{dist}(\operatorname{co} A, B) .
$$


As the element $\xi \in \overline{c o}^{*} A$ is arbitrary, then

$$
\operatorname{dist}\left(\overline{\operatorname{co}}^{*} A, B\right) \leq \operatorname{dist}(\operatorname{co} A, B)
$$

and the necessary equality is proved.

The following propositions are easy to prove.

Proposition 9. The functional $\|\cdot\|_{+}: C_{v}\left(X^{\prime}\right) \cap \mathcal{B}\left(X^{\prime}\right) \rightarrow \mathbb{R}_{+}$defines a norm on $C_{v}\left(X^{\prime}\right) \cap \mathcal{B}\left(X^{\prime}\right)$.

Proposition 10. The functional $\|\cdot\|_{-}: C_{v}\left(X^{\prime}\right) \rightarrow \mathbb{R}_{+}$satisfies the following properties:

(1) $\overline{0} \in A$ if and only if $\|A\|_{-}=0$;

(2) $\|\alpha A\|_{-}=\left|\alpha\|\mid A\|_{-}\right.$, for all $\alpha \in \mathbb{R}$;

(3) $\|A+B\|_{-} \leq\|A\|_{-}+\|B\|_{-}$.

We shall consider a new class of maps of pseudomonotone type. As before $X$ is a Banach space, and let $\langle\cdot, \cdot\rangle_{X}: X^{\prime} \times X \rightarrow \mathbb{R}$ be the duality form.

Definition 11. The multi-valued map $A: X \rightrightarrows X^{\prime}$ is called:

(1) $\lambda$-pseudomonotone, if, for any $\left\{y_{n}\right\}_{n \geq 0} \subset X$ such that $y_{n} \rightarrow y_{0}$ in $X$ from the inequality

$$
\varlimsup_{n \rightarrow \infty}\left\langle d_{n}, y_{n}-y_{0}\right\rangle_{X} \leq 0,
$$

where $d_{n} \in \overline{\mathrm{co}}^{*} A\left(y_{n}\right)$, for all $n \geq 1$, the existence of subsequences $\left\{y_{n_{k}}\right\}_{k \geq 1} \subset$ $\left\{y_{n}\right\}_{n \geq 1}$ and $\left\{d_{n_{k}}\right\}_{k \geq 1} \subset\left\{d_{n}\right\}_{n \geq 1}$ such that

$$
\lim _{k \rightarrow \infty}\left\langle d_{n_{k}}, y_{n_{k}}-w\right\rangle_{X} \geq\left[\overline{\operatorname{co}}^{*} A(y), y_{0}-w\right]_{-} \quad \text { for all } w \in X
$$

follows;

(2) $\lambda_{0}$-pseudomonotone on $X$, if, for any $\left\{y_{n}\right\}_{n \geq 0} \subset X$ such that $y_{n} \rightarrow y_{0}$ in $X$, $d_{n} \rightarrow d_{0}$ in $X^{\prime}$, where $d_{n} \in \overline{\mathrm{co}}^{*} A\left(y_{n}\right)$, for any $n \geq 1$, from the inequality (11) the existence of subsequences $\left\{y_{n_{k}}\right\}_{k \geq 1} \subset\left\{y_{n}\right\}_{n \geq 1}$ and $\left\{d_{n_{k}}\right\}_{k \geq 1} \subset\left\{d_{n}\right\}_{n \geq 1}$, such that (12) holds, follows;

(3) +-coercive, if $\|y\|_{X}^{-1}[A(y), y]_{+} \rightarrow+\infty$ as $\|y\|_{X} \rightarrow+\infty$;

(4) quasi-bounded, if, for all $y_{0} \in X, k_{1}, k_{2}>0$, there exists $N=N\left(k_{1}, k_{2}, y_{0}\right)>0$ such that

for all $y \in X$ such that $\|y\|_{X} \leq k_{1}$, for all $d \in A(y)$ such that $\left\langle d, y-y_{0}\right\rangle_{X} \leq k_{2},\|d\|_{X^{\prime}} \leq N<+\infty$ follows;

(5) bounded, if $A$ converts an arbitrary set bounded in $X$ into a bounded set in $X^{\prime}$; 
(6) monotone, if

$$
\left[A\left(y_{1}\right), y_{1}-y_{2}\right]_{-} \geq\left[A\left(y_{2}\right), y_{1}-y_{2}\right]_{+} \quad \text { for all } y_{1}, y_{2} \in X
$$

(7) a map satisfying the property $(\kappa)$ if for an arbitrary bounded set $D$ in $X$ there exists $c \in \mathbb{R}$ such that

$$
[A(v), v]_{+} \geq c\|v\|_{X} \quad \text { for all } v \in D .
$$

REMARK 12. If $A$ is either bounded, quasi-bounded, or a monotone multi-valued operator (including subdifferential maps), then it satisfies the property $(\kappa)$.

Also, if $A: X \rightarrow C_{v}\left(X^{\prime}\right)$, then in the first two definitions we can replace $d_{n} \in \overline{\mathrm{co}}^{*} A\left(y_{n}\right)$ by $d_{n} \in A\left(y_{n}\right)$, and we can put $A(y)$ instead of $\overline{\mathrm{co}}^{*} A(y)$ in (12).

REMARK 13. Let $A: X \rightrightarrows X^{\prime}$ be +-coercive multi-valued map, and let the multivalued map $F: X \rightrightarrows X^{\prime}$ satisfy the following monotonicity condition:

$$
\left[F\left(y_{1}\right), y_{1}-y_{2}\right]_{+} \geq\left[F\left(y_{2}\right), y_{1}-y_{2}\right]_{-} \quad \text { for all } y_{1}, y_{2} \in X .
$$

Then $A+F: X \rightrightarrows X^{\prime}$ is a + -coercive map.

Indeed, for each $y \in X$,

$$
-[F(0), y]_{-} \leq\|F(0)\|_{-}\|y\|_{X} .
$$

Then, owing to Proposition 6,

$$
\begin{aligned}
{[A(y)+F(y), y]_{+}=} & {[A(y), y]_{+}+[F(y), y]_{+} \geq[A(y), y]_{+} } \\
& +[F(0), y]_{-} \geq[A(y), y]_{+}-\|F(0)\|_{-}\|y\|_{X} .
\end{aligned}
$$

It is clear that $\|F(0)\|_{-}<+\infty$. From here the + -coercivity of the map $A+F$ follows.

REMARK 14. The idea of using subsequences in Definition 11 for single-valued pseudomonotone operators was introduced by Skripnik [19].

REMARK 15. It is obvious that every $\lambda$-pseudomonotone map is $\lambda_{0}$-pseudomonotone. For bounded maps the converse implication is also true. Indeed, let $A: X \rightrightarrows X^{\prime}$ be a $\lambda_{0}$-pseudomonotone map, $y_{n} \rightarrow y$ weakly in $X$, and (11) holds, where $d_{n} \in \overline{\mathrm{co}}^{*} A\left(y_{n}\right)$. From the boundedness of the operator $A$ the boundedness of the operator $\overline{\mathrm{co}}^{*} A$ follows immediately, and then also the boundedness of the sequence $\left\{d_{n}\right\}$ in $X^{\prime}$. Consequently, there exist subsequences $\left\{d_{m}\right\} \subset\left\{d_{n}\right\}$, $\left\{y_{m}\right\} \subset\left\{y_{n}\right\}$, such that $d_{m} \rightarrow d$ weakly star in $X^{\prime}$ and

$$
\varlimsup_{m \rightarrow \infty}\left\langle d_{m}, y_{m}-v\right\rangle_{X} \leq \varlimsup_{n \rightarrow \infty}\left\langle d_{n}, y_{n}-v\right\rangle_{X} \leq 0 .
$$

However, the operator $A$ is $\lambda_{0}$-pseudomonotone. Therefore, passing again to a subsequence,

$$
\varliminf_{m \rightarrow \infty}\left\langle d_{m}, y_{m}-v\right\rangle_{X} \geq\left[\overline{\mathrm{co}}^{*} A(y), y-v\right]_{-} \quad \text { for all } v \in X,
$$

which proves our statement. 
Let us observe that for classical definitions (not passing to subsequences) this statement is problematic.

The concept of a pseudomonotone map has already been extended by Browder and Hess [4], where the class of generalized pseudomonotone operators was defined.

Definition 16. The map $A: X \rightrightarrows X^{\prime}$ is called a generalized pseudomonotone operator, if:

(1) $A(y) \in C_{v}\left(X^{\prime}\right)$ and $A(y)$ is bounded in $X^{\prime}$ for all $y \in D(A)$;

(2) for every pair of sequences $\left\{y_{n}\right\},\left\{d_{n}\right\}$ such that $d_{n} \in A\left(y_{n}\right), y_{n} \rightarrow y$ weakly in $X$, and $d_{n} \rightarrow d$ weakly star in $X^{*}$, satisfying (11), we have $d \in A(y)$ and $\left\langle d_{n}, y_{n}\right\rangle_{X} \rightarrow\langle d, y\rangle_{X}$.

We show now that this class is contained in the class of $\lambda_{0}$-pseudomonotone operators.

PROPOSITION 17. Every generalized pseudomonotone operator is $\lambda_{0^{-}}$ pseudomonotone.

Proof. Let $y_{n} \rightarrow y$ weakly in $X, \overline{\mathrm{co}}^{*} A\left(y_{n}\right) \ni d_{n} \rightarrow d$ weakly star in $X^{\prime}$ and (11) hold. Then, as $A$ is a generalized pseudomonotone operator, $\left\langle d_{n}, y_{n}\right\rangle_{X} \rightarrow\langle d, y\rangle_{X}$, $d \in \overline{\mathrm{co}}^{*} A(y)$. Consequently,

$$
\varliminf_{m \rightarrow \infty}\left\langle d_{n}, y_{n}-v\right\rangle_{X}=\langle d, y-v\rangle_{X} \geq\left[\overline{\mathrm{co}}^{*} A(y), y-v\right]_{-} \quad \text { for all } v \in X .
$$

Proposition 17 is not invertible. Nevertheless, the next statement is true.

Proposition 18. Let $A: X \rightrightarrows X^{\prime}$ be a $\lambda_{0}$-pseudomonotone operator. Then if $y_{n} \rightarrow y$ weakly in $X, \overline{\mathrm{co}}^{*} A\left(y_{n}\right) \ni d_{n} \rightarrow d$ weakly star in $X^{\prime}$ and inequality (11) holds, the existence of subsequences $\left\{y_{m}\right\} \subset\left\{y_{n}\right\},\left\{d_{m}\right\} \subset\left\{d_{n}\right\}$ such that $\left\langle d_{m}, y_{m}\right\rangle_{X} \rightarrow$ $\langle d, y\rangle_{X}$, follows as also does the fact that $d \in \overline{\mathrm{co}}^{*} A(y)$.

Proof. Since $A$ is $\lambda_{0}$-pseudomonotone there exist subsequences $\left\{y_{m}\right\},\left\{d_{m}\right\}$ such that inequality (12) is true. Putting $w=y$, we get $\left\langle d_{m}, y_{m}-y\right\rangle_{X} \rightarrow 0$, so that $\left\langle d_{m}, y_{m}\right\rangle_{X} \rightarrow\langle d, y\rangle_{X}$. Hence,

$$
\langle d, y-v\rangle_{X}=\lim _{m \rightarrow \infty}\left\langle d_{m}, y_{m}-v\right\rangle_{X} \geq\left[\overline{\mathrm{co}}^{*} A(y), y-v\right]_{-} \quad \text { for all } v \in X .
$$

From here and Proposition 6 we obtain $d \in \overline{\mathrm{co}}^{*} A(y)$.

The next lemma is a generalization of the Weierstrass theorem [22].

LEMMA 19. Let $X$ be a Banach space, $K \subset X^{\prime}$ be a weakly star closed set and $L$ : $X^{\prime} \rightarrow \overline{\mathbb{R}}=\mathbb{R} \bigcup\{+\infty\}$ be a weakly star lower semicontinuous functional. Moreover, assume that either the set $K$ is bounded or

$$
\lim _{\|v\|_{X^{\prime}} \rightarrow \infty} L(v)=+\infty
$$


Then the functional $L$ is bounded from below on $K$, reaches on $K$ its minimal lower bound $m$ and the set $E=\{v \in K: L(v)=m\}$ is weakly star compact in $X^{\prime}$.

PROOF. The proof is similar to the proof of Theorem 9.3 in [22].

Proposition 20. Let $A: X \rightrightarrows X^{\prime}$ be a bounded valued $\lambda$-pseudomonotone map. Then, if $y_{n} \rightarrow y$ weakly in $X$ and inequality (11) holds, there exist subsequences $\left\{y_{m}\right\},\left\{d_{m}\right\}$ such that for every $v \in X$ there exists $\zeta(v) \in \overline{\mathrm{co}}^{*} A(y)$, for which

$$
\varliminf_{m \rightarrow \infty}\left\langle d_{m}, y_{m}-v\right\rangle_{X} \geq\langle\zeta(v), y-v\rangle_{X}
$$

Proof. Let $y_{n} \rightarrow y$ weakly in $X, d_{n} \in \overline{\mathrm{co}}^{*} A\left(y_{n}\right)$ and (11) holds. Then, up to subsequences, we come to

$$
\varliminf_{m \rightarrow \infty}\left\langle d_{m}, y_{m}-v\right\rangle_{X} \geq\left[\overline{\mathrm{co}}^{*} A(y), y-v\right]_{-} \quad \text { for all } v \in X
$$

The set $\overline{\mathrm{co}}^{*} A(y)$ is weakly star closed and bounded. Also, the functional defined by $X^{\prime} \ni w \mapsto\langle w, y-v\rangle_{X}$, for all $v \in X$, is weakly star lower semicontinuous. Then from Lemma 19 there exists $\zeta(v) \in \overline{\mathrm{co}}^{*} A(y)$ such that $[A(y), y-v]_{-}=\langle\zeta(v), y-$ $v\rangle_{X}$. Then from inequality (14) we obtain (13).

Definition 21. The multi-valued map $A: X \rightarrow C_{v}\left(X^{\prime}\right)$ satisfies the property $(M)$, if, from $\left\{y_{n}\right\}_{n \geq 0} \subset X, d_{n} \in A\left(y_{n}\right)$, for all $n \geq 1$, and

$$
y_{n} \rightarrow y_{0} \in X, \quad d_{n} \rightarrow d_{0} \in X^{\prime}, \quad \varlimsup_{n \rightarrow \infty}\left\langle d_{n}, y_{n}\right\rangle_{X} \leq\left\langle d_{0}, y_{0}\right\rangle_{X},
$$

it follows that $d_{0} \in A\left(y_{0}\right)$.

Definition 22. The operator $L: D(L) \subset X \rightarrow X^{\prime}$ is said to be maximal monotone if it is monotone and from $\langle w-L(u), v-u\rangle_{X} \geq 0$, for all $u \in D(L)$, it follows that $v \in D(L)$ and $L(v)=w$.

DEFINITION 23. The map $\partial \varphi$ defined by

$$
\partial \varphi(v)=\left\{p \in X^{\prime} \mid\langle p, u-v\rangle_{X} \leq \varphi(u)-\varphi(v) \text { for all } u \in X\right\}
$$

is called the subdifferential map for the convex, lower semicontinuous functional $\varphi: X \rightarrow \mathbb{R}$ at $v \in X$.

It is clear that $\partial \varphi(v) \in C_{v}\left(X^{\prime}\right)$ for all $v \in X$. The fact that $\partial \varphi(v)$ is non-empty is a consequence of the property $\operatorname{int} D(\varphi)=\operatorname{int} D(\partial \varphi)$ (see [2, p. 56]), where $D(\varphi)$ and $D(\partial \varphi)$ are the domains of $\varphi$ and $\partial \varphi$, respectively. Observe that in our case $D(\varphi)=\operatorname{int} D(\varphi)=X$.

Proposition 24. Let $A: X \rightrightarrows X^{\prime}$ be a $\lambda_{0}$-pseudomonotone operator, and let the map $B: X_{\rightarrow}^{\rightarrow} X^{*}$ possess the following properties: 
(1) the map $\overline{\mathrm{co}}^{*} B: X \rightrightarrows X^{\prime}$ is compact, that is the image of a bounded set in $X$ set is precompact in $X^{\prime}$;

(2) the graph of $\overline{\mathrm{co}}^{*} B$ is closed in $X_{w} \times X^{\prime}$ (that is with respect to the weak topology in $X$ and the strong one in $X^{\prime}$ ).

Then the map $C=A+B$ is $\lambda_{0}$-pseudomonotone.

PROOF. Let $y_{n} \rightarrow y$ weakly in $X, d_{n} \in \overline{\mathrm{co}}^{*} C\left(y_{n}\right), d_{n} \rightarrow d$ weakly star in $X^{\prime}$, and

$$
\varlimsup_{n \rightarrow \infty}\left\langle d_{n}, y_{n}-y\right\rangle_{X} \leq 0 .
$$

As the operator $B: X \rightrightarrows X^{\prime}$ is bounded, $\overline{\mathrm{co}}^{*} C=\overline{\mathrm{co}}^{*} A+\overline{\mathrm{co}}^{*} B$. Hence, $d_{n}=d_{n}^{\prime}+d_{n}^{\prime \prime}$, $d_{n}^{\prime} \in \overline{\mathrm{co}}^{*} A\left(y_{n}\right), d_{n}^{\prime \prime} \in \overline{\mathrm{co}}^{*} B\left(y_{n}\right)$. By virtue of the boundedness of $B$, we obtain that $d_{n}^{\prime \prime} \rightarrow d^{\prime \prime}$ weakly star in $X^{\prime}$, so that $d_{n}^{\prime} \rightarrow d^{\prime}=d-d^{\prime \prime}$ weakly star in $X^{\prime}$.

From inequality (11), passing to the subsequence $\left\{y_{m}\right\} \subset\left\{y_{n}\right\}$, we find that

$$
\begin{aligned}
0 & \geq \varlimsup_{n \rightarrow \infty}\left\langle d_{n}, y_{n}-y\right\rangle_{X} \geq \varlimsup_{n \rightarrow \infty}\left\langle d_{n}^{\prime}, y_{n}-y\right\rangle_{X}+\underset{n \rightarrow \infty}{\varliminf_{n \rightarrow \infty}}\left\langle d_{n}^{\prime \prime}, y_{n}-y\right\rangle_{X} \\
& \geq \varlimsup_{m \rightarrow \infty}\left\langle d_{m}^{\prime}, y_{m}-y\right\rangle_{X}+\lim _{m \rightarrow \infty}\left\langle d_{m}^{\prime \prime}, y_{m}-y\right\rangle_{X} .
\end{aligned}
$$

Since $\overline{\mathrm{co}}^{*} B$ is compact and the graph is closed in $X_{w} \times X^{\prime}$, we can consider that $d_{m}^{\prime \prime} \rightarrow d^{\prime \prime}$ strongly in $X^{\prime}$ and, moreover, $d^{\prime \prime} \in \overline{\mathrm{co}}^{*} B(y)$. Then

$$
\varlimsup_{m \rightarrow \infty}\left\langle d_{m}^{\prime}, y_{m}-y\right\rangle_{X} \leq 0 .
$$

Again, passing to subsequences, as $A$ is $\lambda_{0}$-pseudomonotone, we get

$$
\varliminf_{m \rightarrow \infty}\left\langle d_{m}^{\prime}, y_{m}-v\right\rangle_{X} \geq[A(y), y-v]_{-} \quad \text { for all } v \in X,
$$

and then

$$
\begin{aligned}
\varliminf_{m \rightarrow \infty}\left\langle d_{m}, y_{m}-v\right\rangle_{X} & =\underline{\lim }_{m \rightarrow \infty}\left\langle d_{m}^{\prime}, y_{m}-v\right\rangle_{X}+\lim _{m \rightarrow \infty}\left\langle d_{m}^{\prime \prime}, y_{m}-v\right\rangle_{X} \\
& \geq\left[\overline{\mathrm{co}}^{*} A(y), y-v\right]_{-}+\left\langle d^{\prime \prime}, y-v\right\rangle_{X} \\
& \geq\left[\overline{\mathrm{co}}^{*} C(y), y-v\right]_{-} \quad \text { for all } v \in X .
\end{aligned}
$$

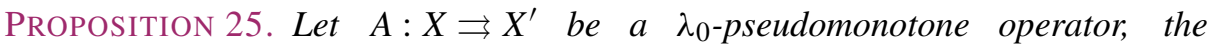
embedding of $X$ in the Banach space $Y$ be compact and dense, and let $\overline{\mathrm{co}}^{*} B: Y_{\rightarrow}^{\rightarrow} Y^{\prime}$ be a locally bounded map such that the graph of $\overline{\mathrm{co}}^{*} B$ is closed in $Y \times Y_{w}^{\prime}$ (that is with respect to the strong topology of $Y$ and the weakly star one in $\left.Y^{\prime}\right)$. Then $C=A+B$ is a $\lambda_{0}$-pseudomonotone map.

PROOF. Let $y_{n} \rightarrow y, d_{n} \in \overline{\mathrm{co}}^{*} C\left(y_{n}\right), d_{n} \rightarrow d$ and let (11) be fulfilled. The operator $\overline{\mathrm{co}}^{*} B$ is locally bounded, that is for all $y \in X$ there exist $N>0$ and $\varepsilon>0$ such that

$$
\left\|\overline{\mathrm{co}}^{*} B(\xi)\right\|_{+} \leq N \quad \text { if }\|\xi-y\|_{X} \leq \varepsilon .
$$


Obviously, a locally bounded operator is bounded-valued. Therefore, $\overline{\mathrm{co}}^{*} C(y)=$ $\overline{\mathrm{co}}^{*} A(y)+\overline{\mathrm{co}}^{*} B(y)$ and $d_{n}=d_{n}^{\prime}+d_{n}^{\prime \prime}, d_{n}^{\prime} \in \overline{\mathrm{co}}^{*} A\left(y_{n}\right), d_{n}^{\prime \prime} \in \overline{\mathrm{co}}^{*} B\left(y_{n}\right)$. Since the imbedding $X \subset Y$ is compact, we have that $y_{n} \rightarrow y$ strongly in $Y$ and by virtue of the local boundedness of $\overline{\mathrm{co}}^{*} B$ the sequence $\left\{d_{n}^{\prime \prime}\right\}$ is bounded in $Y^{\prime}$ (and then also in $X^{\prime}$ ), which means that there will be a subsequence $\left\{d_{m}^{\prime \prime}\right\} \subset\left\{d_{n}^{\prime \prime}\right\}$ such that $d_{m}^{\prime \prime} \rightarrow d^{\prime \prime}$ weakly star in $Y^{\prime}$. The embedding operator $I^{*}: Y^{\prime} \rightarrow X^{\prime}$ is continuous, so that $I^{*}$ remains continuous also in the weakly star topologies [17]. Hence, $d_{m}^{\prime \prime} \rightarrow d^{\prime \prime}$ weakly star in $X^{\prime}$, so that $d_{m}^{\prime}=d_{m}-d_{m}^{\prime \prime} \rightarrow d^{\prime}=d-d^{\prime \prime}$ weakly star in $X^{\prime}$. Therefore

$$
\left\langle d_{m}^{\prime \prime}, y_{m}-y\right\rangle_{X} \rightarrow 0 .
$$

Then from (15) we get $\varlimsup_{m \rightarrow \infty}\left\langle d_{m}^{\prime}, y_{m}-v\right\rangle_{X} \leq 0$, and hence after passing to a subsequence,

$$
\varliminf_{m_{k} \rightarrow \infty}\left\langle d_{m_{k}}^{\prime}, y_{m_{k}}-v\right\rangle_{X} \geq\left[\overline{\mathrm{co}}^{*} A(y), y-v\right]_{-} \quad \text { for all } v \in X .
$$

Furthermore, as the operator $\overline{\mathrm{co}}^{*} B$ is closed in $Y \times Y_{w}^{\prime}$, we have $d^{\prime \prime} \in \overline{\mathrm{co}}^{*} B(y)$ and

$$
\begin{aligned}
\varliminf_{m_{k} \rightarrow \infty}\left\langle d_{m_{k}}, y_{m_{k}}-v\right\rangle_{X} & =\underline{\lim _{k} \rightarrow \infty}\left\langle d_{m_{k}}^{\prime}, y_{m_{k}}-v\right\rangle_{X}+\lim _{m_{k} \rightarrow \infty}\left\langle d_{m_{k}}^{\prime \prime}, y_{m_{k}}-v\right\rangle_{X} \\
& \geq\left[\overline{\mathrm{co}}^{*} A(y), y-v\right]_{-}+\left[\overline{\mathrm{co}}^{*} B(y), y-v\right]_{-} \\
& =\left[\overline{\mathrm{co}}^{*} C(y), y-v\right]_{-} \quad \text { for all } v \in X .
\end{aligned}
$$

Definition 26. The operator $A: X \rightrightarrows X^{\prime}$ is called:

(1) upper radially continuous, if, for all $x, h \in X$,

$$
\varlimsup_{t \rightarrow 0^{+}}[A(x+t h), h]_{+} \leq[A(x), h]_{+} ;
$$

(2) radially semicontinuous, if, for all $x, h \in X$,

$$
\varlimsup_{t \rightarrow 0^{+}}[A(x+t h), h]_{-} \leq[A(x), h]_{+} .
$$

It is obvious that (1) implies (2).

Proposition 27. Let $A: X \rightrightarrows X^{\prime}$ be an upper semicontinuous operator with respect to the strong topology of $X$ and the weakly star topology of $X^{\prime}$. Then $A$ is radially semicontinuous.

Proof. It is well known that $A$ is upper hemicontinuous [1], that is from $x_{n} \rightarrow x$ strongly in $X$ it follows that

$$
\varlimsup_{n \rightarrow \infty}\left[A\left(x_{n}\right), v\right]_{+} \leq[A(x), v]_{+} \text {for all } v \in X .
$$

We note that an upper hemicontinuous operator is upper radially continuous, so that it is radially semicontinuous. 
By $\Pi_{0}$ we will denote the class of continuous functions $C: \mathbb{R}_{+} \times \mathbb{R}_{+} \rightarrow \mathbb{R}$ such that $t^{-1} C\left(r_{1}, t r_{2}\right) \rightarrow 0$ as $t \rightarrow 0^{+}$, for all $r_{1}, r_{2}>0$.

Definition 28. The map $A: X \rightrightarrows X^{\prime}$ is called:

(1) an operator with semibounded variation, if, for any $R>0$ and any $y_{1}, y_{2} \in X$ such that $\left\|y_{i}\right\|_{X} \leq R, i=1,2$, the next inequality holds:

$$
\left[A\left(y_{1}\right), y_{1}-y_{2}\right]_{-} \geq\left[A\left(y_{2}\right), y_{1}-y_{2}\right]_{+}-C\left(R ;\left\|y_{1}-y_{2}\right\|_{X}^{\prime}\right),
$$

where $C \in \Pi_{0}$, and $\|\cdot\|_{X}^{\prime}$ is a compact norm on $X$;

(2) an operator with $l$-semibounded variation, if, instead of (16), the following inequality holds:

$$
\left[A\left(y_{1}\right), y_{1}-y_{2}\right]_{-} \geq\left[A\left(y_{2}\right), y_{1}-y_{2}\right]_{-}-C\left(R ;\left\|y_{1}-y_{2}\right\|_{X}^{\prime}\right) .
$$

Proposition 29. Let $A=A_{0}+A_{1}: X \rightrightarrows X^{\prime}$, where $A_{0}: X \rightrightarrows X^{\prime}$ is a monotone map, and the operator $A_{1}: X \rightrightarrows X^{\prime}$ possesses the following properties:

(1) there is a linear normalized space $Y$ in which $X$ is compactly and densely embedded;

(2) the operator $A_{1}: Y \rightrightarrows Y^{\prime}$ is single-valued and locally polynomial, that is for any $R>0$ there is a natural $n=n(R)$ and a polynom $P_{R}(t)=\sum_{0<\alpha \leq n} \lambda_{\alpha}(R) t^{\alpha}$ with continuous factors $\lambda_{\alpha}(R) \geq 0$ such that the following estimate holds:

$$
\begin{array}{r}
\left\|A_{1}\left(y_{1}\right)-A_{1}\left(y_{2}\right)\right\|_{Y^{\prime}} \leq P_{R}\left(\left\|y_{1}-y_{2}\right\|_{Y}\right) \\
\text { for all }\left\|y_{i}\right\|_{Y} \leq R, i=1,2 .
\end{array}
$$

Then $A$ is an operator with semibounded variation.

Proposition 30. Let the operator $A_{0}: X \rightrightarrows X^{\prime}$ in Proposition 29 satisfy $\left[A_{0}\left(y_{1}\right), y_{1}-y_{2}\right]_{-} \geq\left[A_{0}\left(y_{2}\right), y_{1}-y_{2}\right]_{-}$, for all $y_{1}, y_{2} \in X$ (instead of monotonicity), and let $A_{1}$ satisfy the same conditions. Then $A=A_{0}+A_{1}$ is an operator with l-semibounded variation

PROOF. We prove Proposition 30. For Proposition 29 the arguments are similar.

As $\left[A_{0}\left(y_{1}\right), y_{1}-y_{2}\right]_{-} \geq\left[A_{0}\left(y_{2}\right), y_{1}-y_{2}\right]_{-}$, for all $y_{1}, y_{2} \in X$, by Proposition 6 we need to estimate only $\left[A_{1}\left(y_{1}\right), y_{1}-y_{2}\right]_{-}-\left[A_{1}\left(y_{2}\right), y_{1}-y_{2}\right]_{-}$. We have

$$
\begin{aligned}
& {\left[A_{1}\left(y_{1}\right), y_{1}-y_{2}\right]_{-}-\left[A_{1}\left(y_{2}\right), y_{1}-y_{2}\right]_{-}} \\
& \quad=\left\langle A_{1}\left(y_{1}\right), y_{1}-y_{2}\right\rangle_{X}-\left\langle A_{1}\left(y_{2}\right), y_{1}-y_{2}\right\rangle_{X} \\
& \quad \geq-\left\|A_{1}\left(y_{1}\right)-A_{1}\left(y_{2}\right)\right\|_{Y^{\prime}}\left\|y_{1}-y_{2}\right\|_{Y} .
\end{aligned}
$$

From here and (18) at $\left\|y_{i}\right\|_{X} \leq R(i=1,2)$ (and then $\left\|y_{i}\right\|_{Y} \leq \hat{R}$ ) we obtain

$$
\left[A_{1}\left(y_{1}\right), y_{1}-y_{2}\right]_{-} \geq\left[A_{1}\left(y_{2}\right), y_{1}-y_{2}\right]_{-}-C\left(\hat{R} ;\left\|y_{1}-y_{2}\right\|_{X}^{\prime}\right),
$$

where $\|\cdot\|_{X}^{\prime}=\|\cdot\|_{Y}, C(R, t)=P_{R}(t) t$.

It is easy to check that $C \in \Pi_{0}$. 
PROPOSITION 31. Let one of the following two conditions be satisfied:

(1) $\quad A: X \rightrightarrows X^{\prime}$ is a radially semicontinuous operator with semibounded variation;

(2) $\quad A: X \rightrightarrows X^{\prime}$ is an upper radially continuous operator with l-semibounded variation and compact values.

Then A is a $\lambda_{0}$-pseudomonotone map.

PRoof. Let $y_{n} \rightarrow y$ weakly in $X, \overline{\mathrm{co}}^{*} A\left(y_{n}\right) \ni d_{n} \rightarrow d$ weakly star in $X^{\prime}$ and $\varlimsup_{n \rightarrow \infty}\left\langle d_{n}, y_{n}-y\right\rangle_{X} \leq 0$. Using the fact that $A$ is an operator with semibounded variation we conclude that

$$
\begin{array}{r}
\left\langle d_{n}, y_{n}-v\right\rangle_{X} \geq\left[A\left(y_{n}\right), y_{n}-v\right]_{-} \geq\left[A(v), y_{n}-v\right]_{+}-C\left(R ;\left\|y_{n}-v\right\|_{X}^{\prime}\right) \\
\text { for all } v \in X .
\end{array}
$$

The function $X \ni w \mapsto[A(v), w]_{+}$is convex and semicontinuous from below, and so it is weakly semicontinuous from below. Therefore, substituting in the last inequality $v=y$ and passing to a limit, in view of the properties of the function $C$, we have $\underline{\lim }_{n \rightarrow \infty}\left\langle d_{n}, y_{n}-y\right\rangle_{X} \geq 0$, that is $\left\langle d_{n}, y_{n}-y\right\rangle_{X} \rightarrow 0$.

For any $h \in X$ and $\tau \in[0,1]$ we shall put $w(\tau)=\tau h+(1-\tau) y$. Then

$$
\left\langle d_{n}, y_{n}-w(\tau)\right\rangle_{X} \geq\left[A(w(\tau)), y_{n}-w(\tau)\right]_{+}-C\left(R ;\left\|y_{n}-w(\tau)\right\|_{X}^{\prime}\right)
$$

and

$$
\tau \varliminf_{n \rightarrow \infty}\left\langle d_{n}, y-h\right\rangle_{X} \geq \tau[A(w(\tau)), y-h]_{+}-C\left(R ; \tau\|y-h\|_{X}^{\prime}\right) .
$$

Dividing by $\tau$ and passing to a limit as $\tau \rightarrow 0^{+}$, in view of the radial semicontinuity of $A$ and the properties of the function $C$, we obtain that, for all $h \in X$,

$$
\begin{aligned}
& \underline{\lim }_{n \rightarrow \infty}\left\langle d_{n}, y-h\right\rangle_{X} \geq \underline{\lim }_{\tau \rightarrow 0^{+}}[A(w(\tau)), y-h]_{+}-\lim _{\tau \rightarrow 0^{+}} \frac{1}{\tau} C\left(R ; \tau\|y-h\|_{X}^{\prime}\right) \\
& \geq[A(y), y-h]_{-} \text {. }
\end{aligned}
$$

As $\left\langle d_{n}, y_{n}-y\right\rangle_{X} \rightarrow 0$, then by Proposition 6 we obtain

$$
\begin{aligned}
\underline{\lim }_{n \rightarrow \infty}\left\langle d_{n}, y_{n}-h\right\rangle_{X} & =\underline{\lim _{n \rightarrow \infty}}\left\langle d_{n}, y-h\right\rangle_{X} \geq[A(y), y-h]_{-} \\
& =\left[\overline{\operatorname{co}}^{*} A(y), y-h\right]_{-} \quad \text { for all } h \in X,
\end{aligned}
$$

which proves the first statement of the proposition.

We shall stop now only on the basic distinctive moments of the second statement. Since $A$ is an operator with $l$-semibounded variation, we conclude that

$$
\begin{aligned}
\varliminf_{n \rightarrow \infty}\left\langle d_{n}, y_{n}-v\right\rangle_{X} & \geq \underset{n \rightarrow \infty}{\lim _{n \rightarrow \infty}}\left[A\left(y_{n}\right), y_{n}-v\right]_{-} \\
& \geq \underset{n \rightarrow \infty}{\lim _{n \rightarrow \infty}}\left[A(v), y_{n}-v\right]_{-}-C\left(R ;\|y-v\|_{X}^{\prime}\right) .
\end{aligned}
$$


Let us estimate the first term on the right-hand side of (19). We shall prove that the function $X \ni h \mapsto[A(v), h]_{-}$is weakly semicontinuous from below for all $v \in X$. Let $z_{n} \rightarrow z$ weakly in $X$. For any $n=1,2, \ldots$ there exists $\xi_{n} \in \overline{\mathrm{co}}^{*} A(v)$ such that $\left[A(v), z_{n}\right]_{-}=\left\langle\xi_{n}, z_{n}\right\rangle_{X}$. From the sequence $\left\{\xi_{n} ; z_{n}\right\}$ we can choose a subsequence $\left\{\xi_{m} ; z_{m}\right\}$ such that

$$
\varliminf_{n \rightarrow \infty}\left[A(v), z_{n}\right]_{-}=\varliminf_{n \rightarrow \infty}\left\langle\xi_{n}, z_{n}\right\rangle_{X}=\lim _{m \rightarrow \infty}\left\langle\xi_{m}, z_{m}\right\rangle_{X},
$$

and by virtue of the compactness of set $\overline{\mathrm{co}}^{*} A(v)$ we can conclude that $\xi_{m} \rightarrow \xi$ strongly in $X^{\prime}$, and that $\xi \in \overline{\mathrm{co}}^{*} A(v)$. Hence

$$
\varliminf_{n \rightarrow \infty}\left[A(v), z_{n}\right]_{-}=\lim _{m \rightarrow \infty}\left\langle\xi_{m}, z_{m}\right\rangle_{X}=\langle\xi, z\rangle_{X} \geq[A(v), z]_{-},
$$

which proves the weak semicontinuity from below of the map $h \mapsto[A(v), h]_{-}$.

In that case from (19) we obtain

$$
\varliminf_{n \rightarrow \infty}\left\langle d_{n}, y_{n}-v\right\rangle_{X} \geq \varliminf_{n \rightarrow \infty}^{\lim _{n}}\left[A\left(y_{n}\right), y_{n}-v\right]_{-} \geq[A(v), y-v]_{-}-C\left(R ;\|y-v\|_{X}^{\prime}\right),
$$

where, substituting $v=y$, we have $\left\langle d_{n}, y_{n}-y\right\rangle_{X} \rightarrow 0$. Therefore

$$
\lim _{n \rightarrow \infty}\left\langle d_{n}, y-v\right\rangle_{X} \geq[A(v), y-v]_{-}-C\left(R ;\|y-v\|_{X}^{\prime}\right) \quad \text { for all } v \in X .
$$

Substituting in the last inequality $v=t w+(1-t) y, w \in X, t \in[0,1]$, dividing the result by $t$ and passing to the limit as $t \rightarrow 0^{+}$, in view of the upper radial continuity we find

$$
\varliminf_{n \rightarrow \infty}\left\langle d_{n}, y_{n}-w\right\rangle_{X} \geq[A(y), y-w]_{-}=\left[\overline{\mathrm{co}}^{*} A(y), y-w\right]_{-} \quad \text { for all } w \in X
$$

We shall now define the space $S\left(X ; X^{\prime}\right)$. We denote by $S\left(X ; X^{\prime}\right)$ the family of all multi-valued maps from the Banach $X$ into $X^{\prime}$. Let us consider the partial order on the set $S\left(X ; X^{\prime}\right)$ defined by

$$
F \subset G \text { if and only if } F(x) \subset G(x) \text { for all } x \in X .
$$

Let us consider the following operations.

(I) For every $A, B \in S\left(X ; X^{\prime}\right)$ there is a unique map $C \in S\left(X ; X^{\prime}\right)$, which is called the sum of $A$ and $B(C=A+B)$, defined by the rule

$$
C(y)=A(y)+B(y)=\{a+b \mid a \in A(y), b \in B(y)\} .
$$

Moreover, the following properties hold:

(a) $A+B=B+A$ (commutativity);

(b) $A+(B+C)=(A+B)+C$ (associativity); 
(c) there is an element $0 \in S\left(X ; X^{\prime}\right)$ such that $A+0=A$, for all $A \in S\left(X ; X^{\prime}\right)$

(d) for each $A \in S\left(X ; X^{\prime}\right)$ there exists an element $(-A) \in S\left(X ; X^{\prime}\right)$ such that $A+(-A) \geq 0$ and $-(-A)=A$.

(II) For each real number $\alpha$ and for each element $A \in S\left(X ; X^{\prime}\right)$ an element $\alpha A \in$ $S\left(X ; X^{\prime}\right)$ is well defined by the rule

$$
(\alpha A)(y)=\alpha(A(y))=\{\alpha a \mid a \in A(y)\} .
$$

The following properties are satisfied:
(a) $\quad \alpha(\beta A)=(\alpha \beta) A$
(b) $1 \cdot A=A$, for all $A \in S\left(X ; X^{\prime}\right)$;
(c) $(\alpha+\beta) A \leq \alpha A+\beta A$;
(d) $\quad \alpha(A+B)=\alpha A+\alpha B$.

Note that the space $S\left(X ; X^{\prime}\right)$ is not a linear space concerning the operations given above.

Definition 32. The multi-valued maps $A: V \rightrightarrows V^{\prime}$ and $B: W \rightrightarrows W^{\prime}$ are called $s$-mutually bounded if for any $M>0$ there exists $K(M)>0$ such that from $\|y\|_{X} \leq M$ and $\left\langle d_{1}(y)+d_{2}(y), y\right\rangle \leq M$ we have either $\left\|d_{1}(y)\right\|_{V^{\prime}} \leq K(M)$ or $\left\|d_{2}(y)\right\|_{W^{\prime}} \leq K(M)$. Here $d_{1}$ and $d_{2}$ are arbitrary selectors of the maps $A$ and $B$, respectively.

LEMMA 33. Let $V, W$ be Banach spaces, densely and continuously embedded in the locally convex linear topological space $Y$. We have the following results.

(1) Let $A: V \rightrightarrows V^{\prime}, B: W \rightrightarrows W^{\prime}$ be multi-valued $\lambda$-pseudomonotone maps and one of them be bounded-valued. Then the multi-valued operator $C:=A+B$ : $V \cap W:=X \rightrightarrows V^{\prime}+W^{\prime}$ is $\lambda$-pseudomonotone.

(2) Let $A: V \rightrightarrows V^{\prime}, B: W \rightrightarrows W^{\prime}$ be multi-valued $\lambda_{0}$-pseudomonotone maps, let one of them be bounded-valued, the pair $(A ; B)$ be s-mutually bounded and the spaces $V$ and $W$ be reflexive. Then $C:=A+B: V \cap W:=X \rightrightarrows V^{\prime}+W^{\prime}$ is $\lambda_{0}$-pseudomonotone.

REMARK 34. Lemma 33 means that the family of all $\lambda$-pseudomonotone multivalued maps on $X$ with bounded values is a convex cone in the space $S\left(X ; X^{\prime}\right)$.

REMARK 35. Obviously, if one of the operators from the pair $(A ; B)$ is bounded, then the pair $(A ; B)$ is $s$-mutually bounded.

Proof. Let $y_{n} \rightarrow y$ in $X$ (that is, $y_{n} \rightarrow y$ in $V$ and $y_{n} \rightarrow y$ in $W$ ) and let inequality (11) hold with

$$
d_{n} \in \overline{\mathrm{co}}^{*} C\left(y_{n}\right)=\overline{\mathrm{co}}^{*} A\left(y_{n}\right)+\overline{\mathrm{co}}^{*} B\left(y_{n}\right) .
$$

The last equality follows from the fact that one of the maps is boundedvalued. Consequently, $d_{n}=d_{n}^{\prime}+d_{n}^{\prime \prime}$, where $d_{n}^{\prime} \in \overline{\operatorname{co}}^{*} A\left(y_{n}\right), \quad d_{n}^{\prime \prime} \in \overline{\operatorname{co}}^{*} B\left(y_{n}\right)$. 
From inequality (11)

$$
\lim _{n \rightarrow \infty}\left\langle d_{n}^{\prime \prime}, y_{n}-y_{0}\right\rangle_{W}+\varlimsup_{n \rightarrow \infty}\left\langle d_{n}^{\prime}, y_{n}-y_{0}\right\rangle_{V} \leq \varlimsup_{n \rightarrow \infty}\left\langle d_{n}, y_{n}-y_{0}\right\rangle_{X} \leq 0,
$$

or, symmetrically,

$$
\lim _{n \rightarrow \infty}\left\langle d_{n}^{\prime}, y_{n}-y_{0}\right\rangle_{V}+\varlimsup_{n \rightarrow \infty}\left\langle d_{n}^{\prime \prime}, y_{n}-y_{0}\right\rangle_{W} \leq \varlimsup_{n \rightarrow \infty}\left\langle d_{n}, y_{n}-y_{0}\right\rangle_{X} \leq 0 .
$$

In the case of the second statement we have to obtain additionally that, up to subsequences,

$$
d_{n}^{\prime} \rightarrow d_{0}^{\prime} \text { in } V^{\prime} \quad \text { and } \quad d_{n}^{\prime \prime} \rightarrow d_{0}^{\prime \prime} \text { in } W^{\prime} .
$$

As the pair $(A ; B)$ is $s$-mutually bounded, from the estimate $\left\langle d_{n}(y), y\right\rangle_{X}=\left\langle d_{n}^{\prime}(y)+\right.$ $\left.d_{n}^{\prime \prime}(y), y\right\rangle_{X} \leq M$ we have either $\left\|d_{n}^{\prime}(y)\right\|_{V^{\prime}} \leq K(M)$ or $\left\|d_{n}^{\prime \prime}(y)\right\|_{W^{\prime}} \leq K(M)$. Thus, (21) follows.

Further, without loss of generality, let us consider inequality (20). Obviously there exist subsequences $\left\{y_{n_{k}}\right\}_{k \geq 1} \subset\left\{y_{n}\right\}_{n \geq 1}$ and $\left\{d_{n_{k}}\right\}_{k \geq 1} \subset\left\{d_{n}\right\}_{n \geq 1}$ for which

$$
\begin{aligned}
& \lim _{n \rightarrow \infty}\left\langle d_{n}^{\prime}, y_{n}-y_{0}\right\rangle_{V}+\varlimsup_{n \rightarrow \infty}\left\langle d_{n}^{\prime \prime}, y_{n}-y_{0}\right\rangle_{W} \\
& \quad \geq \lim _{k \rightarrow \infty}\left\langle d_{n_{k}}^{\prime}, y_{n_{k}}-y_{0}\right\rangle_{V}+\varlimsup_{k \rightarrow \infty}\left\langle d_{n_{k}}^{\prime \prime}, y_{n_{k}}-y_{0}\right\rangle_{W} .
\end{aligned}
$$

From here, up to subsequences, we shall obtain one of the following two inequalities:

$$
\varlimsup_{n \rightarrow \infty}\left\langle d_{n}^{\prime}, y_{n}-y\right\rangle_{V} \leq 0 \quad \text { or } \varlimsup_{n \rightarrow \infty}\left\langle d_{n}^{\prime \prime}, y_{n}-y\right\rangle_{W} \leq 0 .
$$

Without loss of generality, let us consider that (up to a subsequence) $\varlimsup_{n \rightarrow \infty}\left\langle d_{n}^{\prime}\right.$, $\left.y_{n}-y\right\rangle_{V} \leq 0$. Then, owing to the $\lambda$-pseudomonotonicity of $A$ there exists $\left\{y_{m}\right\}_{m} \subset$ $\left\{y_{n}\right\}_{n}$ such that

$$
\lim _{m \rightarrow \infty}\left\langle d_{m}^{\prime}, y_{m}-v\right\rangle_{V} \geq\left[\overline{\mathrm{co}}^{*} A(y), y-v\right]_{-} \quad \text { for all } v \in V .
$$

We put in the last inequality $v=y$. Then

$$
\lim _{m \rightarrow \infty}\left\langle d_{m}^{\prime}, y_{m}-y\right\rangle_{V} \geq\left[\overline{\mathrm{co}}^{*} A(y), y-y\right]_{-}=0 .
$$

Hence, there exists $\lim _{m \rightarrow \infty}\left\langle d_{m}^{\prime}, y_{m}-y\right\rangle_{V}=0$. Then, due to (11), $\varlimsup_{n \rightarrow \infty}\left\langle d_{m}^{\prime \prime}\right.$, $\left.y_{m}-y\right\rangle_{W} \leq 0$. In view of (22) and the $\lambda$-pseudomonotonicity of $A$ and $B$ there exist $\left\{y_{n_{k}}\right\}_{k \geq 1} \subset\left\{y_{n}\right\}_{n \geq 1}$ and $\left\{d_{n_{k}}\right\}_{k \geq 1} \subset\left\{d_{n}\right\}_{n \geq 1}$ such that

$$
\begin{array}{ll}
\lim _{k \rightarrow \infty}\left\langle d_{n_{k}}^{\prime}, y_{n_{k}}-v\right\rangle_{V} \geq\left[\overline{\mathrm{co}}^{*} A(y), y-v\right]_{-} & \text {for all } v \in V, \\
\lim _{k \rightarrow \infty}\left\langle d_{n_{k}}^{\prime \prime}, y_{n_{k}}-w\right\rangle_{W} \geq\left[\overline{\mathrm{co}}^{*} B(y), y-w\right]_{-} & \text {for all } w \in W .
\end{array}
$$


Then from last two relations it follows that

$$
\begin{aligned}
\lim _{k \rightarrow \infty}\left\langle d_{n_{k}}, y_{n_{k}}-x\right\rangle_{X} & \geq \lim _{k \rightarrow \infty}\left\langle d_{n_{k}}^{\prime}, y_{n_{k}}-x\right\rangle_{V}+\lim _{k \rightarrow \infty}\left\langle d_{n_{k}}^{\prime \prime}, y_{n_{k}}-x\right\rangle_{W} \\
& \geq[A(y), y-x]_{-}+[B(y), y-x]_{-} \\
& =[C(y), y-x]_{-}=\left[\overline{\operatorname{co}}^{*} C(y), y-x\right]_{-} \quad \text { for all } x \in V \cap W
\end{aligned}
$$

The lemma is proved.

LEMMA 36. Let $V, W$ be Banach spaces, densely and continuously embedded in the locally convex linear topological space $Y$, and let $A: V \rightrightarrows V^{\prime}, B: W_{\rightarrow} W^{\prime}$ be multi-valued +-coercive maps, which satisfy condition $(\kappa)$ (see Definition 11). Then the multi-valued operator $C:=A+B: V \cap W \rightrightarrows V^{\prime}+W^{\prime}$ is +-coercive.

PROOF. We prove the lemma by contradiction. Let us assume that there exist $\left\{x_{n}\right\}_{n \geq 1}$ such that $\left\|x_{n}\right\|_{X}=\left\|x_{n}\right\|_{V}+\left\|x_{n}\right\|_{W} \rightarrow+\infty$ as $n \rightarrow \infty$, but $\sup _{n \geq 1}\left[\left[C\left(x_{n}\right), x_{n}\right]_{+} /\left\|x_{n}\right\|_{X}\right]<+\infty$.

CASE 1. Let $\left\|x_{n}\right\|_{V} \rightarrow+\infty$ as $n \rightarrow \infty,\left\|x_{n}\right\|_{W} \leq c$, for all $n \geq 1$. Denote

$$
\gamma_{A}(r):=\inf _{\|v\|_{V}=r} \frac{[A(v), v]_{+}}{\|v\|_{V}}, \quad \gamma_{B}(r):=\inf _{\|w\|_{W}=r} \frac{[B(w), w]_{+}}{\|w\|_{W}}, \quad r>0 .
$$

Note that $\gamma_{A}(r) \rightarrow+\infty, \gamma_{B}(r) \rightarrow+\infty$ as $r \rightarrow+\infty$. Then for any $n \geq 1$ we have $\left\|x_{n}\right\|_{V}^{-1}\left[A\left(x_{n}\right), x_{n}\right]_{+} \geq \gamma_{A}\left(\left\|x_{n}\right\|_{V}\right)$ and

$$
\frac{\left[A\left(x_{n}\right), x_{n}\right]_{+}}{\left\|x_{n}\right\|_{X}} \geq \gamma_{A}\left(\left\|x_{n}\right\|_{V}\right) \frac{\left\|x_{n}\right\|_{V}}{\left\|x_{n}\right\|_{X}} \rightarrow+\infty \quad \text { as }\left\|x_{n}\right\|_{V} \rightarrow+\infty \text { and }\left\|x_{n}\right\|_{W} \leq c .
$$

In this case, in view of condition $(\kappa)$, for all $n \geq 1$ one has

$$
\frac{\left[B\left(x_{n}\right), x_{n}\right]_{+}}{\left\|x_{n}\right\|_{X}} \geq \gamma_{B}\left(\left\|x_{n}\right\|_{W}\right) \frac{\left\|x_{n}\right\|_{W}}{\left\|x_{n}\right\|_{X}} \geq c_{1} \frac{\left\|x_{n}\right\|_{W}}{\left\|x_{n}\right\|_{X}} \rightarrow 0 \quad \text { as } n \rightarrow \infty,
$$

where $c_{1} \in \mathbb{R}$ is a constant from condition $(\kappa)$. It is clear that

$$
\frac{\left[C\left(x_{n}\right), x_{n}\right]_{+}}{\left\|x_{n}\right\|_{X}}=\frac{\left[A\left(x_{n}\right), x_{n}\right]_{+}}{\left\|x_{n}\right\|_{X}}+\frac{\left[B\left(x_{n}\right), x_{n}\right]_{+}}{\left\|x_{n}\right\|_{X}} \rightarrow+\infty \quad \text { as } n \rightarrow \infty .
$$

We have a contradiction with the boundedness of the left-hand side of the given expression.

CASE 2. The case $\left\|x_{n}\right\|_{V} \leq c$, for all $n \geq 1$, and $\left\|x_{n}\right\|_{W} \rightarrow \infty$ as $n \rightarrow \infty$ is proved similarly. 
CASE 3. Let us consider the situation where $\left\|x_{n}\right\|_{V} \rightarrow \infty$ and $\left\|x_{n}\right\|_{W} \rightarrow \infty$ as $n \rightarrow \infty$. Then,

$$
\begin{aligned}
+\infty>\sup _{n \geq 1} \frac{\left[C\left(x_{n}\right), x_{n}\right]_{+}}{\left\|x_{n}\right\|_{X}} \geq & \gamma_{A}\left(\left\|x_{n}\right\|_{V}\right) \frac{\left\|x_{n}\right\|_{V}}{\left\|x_{n}\right\|_{V}+\left\|x_{n}\right\|_{W}} \\
& +\gamma_{B}\left(\left\|x_{n}\right\|_{W}\right) \frac{\left\|x_{n}\right\|_{W}}{\left\|x_{n}\right\|_{V}+\left\|x_{n}\right\|_{W}} .
\end{aligned}
$$

It is obvious that, for any $n \geq 1,\left\|x_{n}\right\|_{V} /\left\|x_{n}\right\|_{X}>0$ and $\left\|x_{n}\right\|_{W} /\left\|x_{n}\right\|_{X}>0$. If, for example, $\left\|x_{n}\right\|_{V} /\left\|x_{n}\right\|_{X} \rightarrow 0$, then $\left\|x_{n}\right\|_{W} /\left\|x_{n}\right\|_{X}=1-\left\|x_{n}\right\|_{V} /\left\|x_{n}\right\|_{X} \rightarrow 1$. We have a contradiction with (23). Thus, the lemma is proved.

\section{Main result}

In this section we shall prove the existence of solutions of the problem (3)-(4).

THEOREM 37. Assume the following conditions:

(1) $A: \mathcal{V}_{1} \rightarrow C_{v}\left(\mathcal{V}_{1}^{\prime}\right)$ is a bounded, $\lambda$-pseudomonotone on $\mathcal{V}_{1}$ operator, which satisfies the + -coerciveness condition on $\mathcal{V}_{1}$;

(2) the functional $\varphi: \mathcal{V}_{2} \rightarrow \mathbb{R}$ is convex, lower semicontinuous and satisfies the following coerciveness condition:

$$
\frac{\varphi(v)}{\|v\| \mathcal{V}_{2}} \longrightarrow+\infty \quad \text { as }\|v\| \mathcal{V}_{2} \longrightarrow+\infty
$$

(3) the operator $\Lambda$ satisfies all the conditions given in (7)-(10).

Then for any $f \in \mathcal{V}^{\prime}$ there exists $u \in \mathcal{V}$ satisfying (3)-(4).

REMARK 38. If $\mathcal{V} \subset \mathcal{H}$ with dense and continuous embedding, inclusion (3) and Remark 4 imply that $u \in \mathcal{V} \cap D\left(\Lambda ; \mathcal{V}^{\prime}\right)$.

PROOF. Let us consider the more general problem

$$
\begin{gathered}
u \in D\left(\Lambda ; \mathcal{V}, \mathcal{V}^{\prime}\right), \\
\Lambda u+\mathcal{A}(u)+\mathcal{B}(u) \ni f,
\end{gathered}
$$

where $\mathcal{B}: \mathcal{V}_{2} \rightarrow C_{v}\left(\mathcal{V}_{2}^{\prime}\right)$ is a $\lambda$-pseudomonotone on $\mathcal{V}_{2}$, quasi-bounded multi-valued operator, which satisfies the + -coerciveness condition on $\mathcal{V}_{2}$.

Let us prove first that $\mathcal{B}:=\partial \varphi: \mathcal{V}_{2} \rightarrow C_{v}\left(\mathcal{V}_{2}^{\prime}\right)$ satisfies these properties.

Quasi-boundedness. Let $y_{0} \in \mathcal{V}_{2}, k_{1}, k_{2}>0$ be arbitrary fixed elements. Then, for any $y \in \mathcal{V}_{2}$ and $d \in \partial \varphi(y)$ such that $\|y\|_{\mathcal{V}_{2}} \leq k_{1}$ and $\left\langle d, y-y_{0}\right\rangle \leq k_{2}$, we have

$$
\begin{aligned}
\langle d, u\rangle_{\mathcal{V}_{2}} & =\left\langle d, u+y_{0}-y\right\rangle_{\mathcal{V}_{2}}+\left\langle d, y-y_{0}\right\rangle_{\mathcal{V}_{2}} \\
& \leq \varphi\left(u+y_{0}\right)-\varphi(y)+k_{2} \leq \varphi\left(u+y_{0}\right)-\inf _{y \in\left(\bar{B}_{k_{1}}\right)} \varphi(y)+k_{2} \\
& =\text { Constant }<+\infty,
\end{aligned}
$$


as a convex lower semicontinuous functional is bounded from below on bounded sets. Hence, by the Banach-Steinhaus theorem, there exists $N=N\left(y_{0}, k_{1}, k_{2}\right)$ such that $\|d\|_{\mathcal{V}_{2}^{\prime}} \leq N$. Here, $\bar{B}_{k_{1}}$ denotes a closed ball of radius $k_{1}$ centered at 0 .

+ -coerciveness on $\mathcal{V}_{2}$. Let us put $u=\overline{0}$ in Definition 23. Then

$$
\|y\|_{\mathcal{V}_{2}}^{-1}[\partial \varphi(y), y]_{+} \geq\|y\|_{\mathcal{V}_{2}}^{-1} \varphi(y)-\|y\|_{\mathcal{V}_{2}}^{-1} \varphi(\overline{0}) \rightarrow+\infty \quad \text { as }\|y\| \mathcal{V}_{2} \rightarrow+\infty
$$

$\lambda$-pseudomonotonicity on $\mathcal{V}_{2}$. Let $y_{n} \rightarrow y_{0}$ in $\mathcal{V}_{2}$ and let inequality (11) hold for $d_{n} \in \partial \varphi\left(y_{n}\right), n \geq 1$. Then, in view of the quasi-boundedness of $\partial \varphi$ on $\mathcal{V}_{2}$, it follows that $\left\{d_{n}\right\}_{n \geq 1}$ is bounded in $\mathcal{V}_{2}^{\prime}$. Hence, there exists a subsequence $\left\{d_{n_{k}}\right\}_{k \geq 1} \subset\left\{d_{n}\right\}_{n \geq 1}$ such that $\bar{d}_{n_{k}} \rightarrow d$ in $\mathcal{V}_{2}^{\prime}$. On the other hand, due to the monotonicity of $\partial \varphi$, for any $d_{0} \in \partial \varphi\left(y_{0}\right)$, for all $k \geq 1$,

$$
\left\langle d_{n_{k}}, y_{n_{k}}-y_{0}\right\rangle_{\mathcal{V}_{2}}=\left\langle d_{n_{k}}-d_{0}, y_{n_{k}}-y_{0}\right\rangle_{\mathcal{V}_{2}}+\left\langle d_{0}, y_{n_{k}}-y_{0}\right\rangle_{\mathcal{V}_{2}} \geq\left\langle d_{0}, y_{n_{k}}-y_{0}\right\rangle \mathcal{V}_{2} .
$$

Then $\underline{\lim }_{k \rightarrow+\infty}\left\langle d_{n_{k}}, y_{n_{k}}-y_{0}\right\rangle_{\mathcal{V}_{2}} \geq \underline{\lim }_{k \rightarrow+\infty}\left\langle d_{0}, y_{n_{k}}-y_{0}\right\rangle_{\mathcal{V}_{2}}=0$, which together with (11) gives

$$
\lim _{k \rightarrow+\infty}\left\langle d_{n_{k}}, y_{n_{k}}-y_{0}\right\rangle_{\mathcal{V}_{2}}=0
$$

Thus, for all $w \in \mathcal{V}_{2}$,

$$
\begin{aligned}
\lim _{k \rightarrow+\infty}\left\langle d_{n_{k}}, y_{n_{k}}-w\right\rangle_{\mathcal{V}_{2}} & \geq \lim _{k \rightarrow+\infty}\left\langle d_{n_{k}}, y_{n_{k}}-y_{0}\right\rangle_{\mathcal{V}_{2}}+\frac{\lim _{k \rightarrow+\infty}\left\langle d_{n_{k}}, y_{0}-w\right\rangle_{\mathcal{V}_{2}}}{} \\
& =\left\langle d_{0}, y_{0}-w\right\rangle \mathcal{V}_{2} .
\end{aligned}
$$

On the other hand,

$$
\begin{aligned}
\left\langle d_{0}, w-y_{0}\right\rangle_{\mathcal{V}_{2}} & \leq \overline{\lim _{k \rightarrow+\infty}}\left\langle d_{n_{k}}, w-y_{0}\right\rangle_{\mathcal{V}_{2}} \\
& \leq \varphi(w)-\frac{\lim }{k \rightarrow+\infty} \varphi\left(y_{n_{k}}\right) \\
& \leq \varphi(w)-\varphi\left(y_{0}\right)
\end{aligned}
$$

because a convex lower semicontinuous function is weakly lower semicontinuous. From (27) and Definition 23 it follows that $d_{0} \in \partial \varphi\left(y_{0}\right)$. From here, thanks to Proposition 6 and (26), we get inequality (12) for $A=\partial \varphi$ on $\mathcal{V}_{2}$.

Theorem 37 is then a direct consequence of the following result.

THEOREM 39. For any $f \in \mathcal{V}^{\prime}$ there exists $u \in \mathcal{V}$ satisfying (24)-(25).

PROOF. Let us use the coercivity condition. From Lemma 36 and Remark 12 it follows that $\mathcal{A}+\mathcal{B}$ is + -coercive on $\mathcal{V}$. Hence, there exists $R>0$ such that, for all $u \in \mathcal{V}$ satisfying $\|u\| \mathcal{V}=R$, we get

$$
[\mathcal{A}(u)+\mathcal{B}(u), u]_{+} \geq 0 .
$$


We shall now consider approximative solutions. The natural approximation of inclusion (25) is the inclusion

$$
\frac{I-G(h)}{h} u_{h}+\mathcal{A}\left(u_{h}\right)+\mathcal{B}\left(u_{h}\right) \ni f \quad(h>0) .
$$

However, if $\mathcal{V}$ is not contained in $\mathcal{H}$, then (29), generally speaking, has no solutions, and it is necessary to modify the given inclusion in an appropriate way. We shall choose a sequence $\theta_{h} \in(0,1)$ such that

$$
\frac{1-\theta_{h}}{h} \rightarrow 0 \quad \text { as } h \rightarrow 0 .
$$

We put $\theta_{h}=1$ if $\mathcal{V} \subset \mathcal{H}$. Further, we define

$$
\Lambda_{h}=\frac{I-\theta_{h} G(h)}{h},
$$

and replace (29) by the inclusion

$$
\Lambda_{h} u_{h}+\mathcal{A}\left(u_{h}\right)+\mathcal{B}\left(u_{h}\right) \ni f .
$$

LEMMA 40. Inclusion (31) has a solution $u_{h} \in \mathcal{V} \cap \mathcal{H}$ such that $\left\|u_{h}\right\|_{\mathcal{V}} \leq R$.

PROOF. Let us consider the map

$$
\mathcal{D}_{h}=\Lambda_{h}+\mathcal{A}: \mathcal{H} \cap \mathcal{V}_{1} \rightarrow C_{v}\left(\mathcal{H}+\mathcal{V}_{1}^{\prime}\right),
$$

and also the following inclusion:

$$
\mathcal{D}_{h}\left(u_{h}\right)+\mathcal{B}\left(u_{h}\right) \ni f .
$$

The existence of a solution $u_{h} \in \mathcal{V} \cap \mathcal{H}$ of this inclusion such that $\left\|u_{h}\right\|_{\mathcal{V}} \leq R$ follows from [13, Theorem 2.1] with $V=\mathcal{H} \cap \mathcal{V}_{1}, W=\mathcal{V}_{2}, A=D_{h}, B=\mathcal{B}, L \equiv \overline{0}$, $D(L)=V, f=f, R=R$, and the following lemma.

LEMMA 41. The operator $\mathcal{D}_{h}$ satisfies the following conditions:

$$
\begin{gathered}
{\left[\mathcal{D}_{h}(u)+\mathcal{B}(u), u\right]_{+} \geq 0 \text { for all } u \in \mathcal{V} \text { such that }\|u\| \mathcal{V}=R} \\
\mathcal{D}_{h} \text { is } \lambda \text {-pseudomonotone on } \mathcal{H} \cap \mathcal{V}_{1} \\
\mathcal{D}_{h} \text { is bounded on } \mathcal{H} \cap \mathcal{V}_{1}
\end{gathered}
$$

Proof. As $G(s)$ is non-expansive on $\mathcal{H}$, it follows that, for any $v \in \mathcal{H}$,

$$
\begin{aligned}
\left(\Lambda_{h} v, v\right)_{\mathcal{H}} & =\frac{1}{h}\left(v-\theta_{h} G(h) v, v\right) \geq \frac{1}{h}\left(\|v\|_{\mathcal{H}}^{2}-\theta_{h}\|G(h) v\|_{\mathcal{H}}\|v\|_{\mathcal{H}}\right) \\
& \geq \frac{1-\theta_{h}}{h}\|v\|_{\mathcal{H}}^{2} .
\end{aligned}
$$

From here the +-coercitivity for $\Lambda_{h}$ on $\mathcal{H}$ follows.

Using (28), (35) and Proposition 6, we obtain (32). 
For (34) note that the boundedness of $\mathcal{D}_{h}$ on $\mathcal{H} \cap \mathcal{V}_{1}$ follows from the boundedness of $\Lambda_{h}$ on $\mathcal{H}$ and the boundedness of $\mathcal{A}$ on $\mathcal{V}_{1}$. The boundedness of $\Lambda_{h}$ on $\mathcal{H}$ follows immediately from the definition of $\Lambda_{h}$ and estimate (7). Hence, form Remark 12 it also follows that $\mathcal{D}_{h}$ satisfies the property $(\kappa)$.

Finally, let us prove the $\lambda$-pseudomonotonicity of $D_{h}$ on $\mathcal{H} \cap \mathcal{V}_{1}$. For this purpose Lemma 33 is used with $A=\Lambda_{h}$ on $\mathcal{V}=\mathcal{H}$ and $B=\mathcal{A}$ on $W=\mathcal{V}_{1}$. From here, since $\mathcal{A}$ is $\lambda$-pseudomonotone and has bounded values on $\mathcal{V}_{1}$, it is enough to prove the $\lambda$-pseudomonotonicity of $\Lambda_{h}$ on $\mathcal{H}$. Let us prove it. Indeed, let

$$
y_{n} \rightarrow y \text { in } \mathcal{H}, \quad \varlimsup_{n \rightarrow \infty}\left(\Lambda_{h} y_{n}, y_{n}-y\right)_{\mathcal{H}} \leq 0 .
$$

Then, from estimate (35), we have

$\lim _{n \rightarrow \infty}\left(\Lambda_{h} y_{n}, y_{n}-y\right)_{\mathcal{H}} \geq \lim _{n \rightarrow \infty}\left(\Lambda_{h} y_{n}-\Lambda_{h} y, y_{n}-y\right)_{\mathcal{H}}+\lim _{n \rightarrow \infty}\left(\Lambda_{h} y, y_{n}-y\right)_{\mathcal{H}} \geq 0$

Hence, $\lim _{n \rightarrow \infty}\left(\Lambda_{h} y_{n}, y_{n}-y\right)_{\mathcal{H}}=0$. Further, for any $u \in \mathcal{H}, \quad s>0$, let $w:=y+s(u-y)$. Then from

$$
\begin{aligned}
\left(\Lambda_{h} w-\Lambda_{h} y_{n}, y_{n}-y\right)_{\mathcal{H}} & =\left(\Lambda_{h} w-\Lambda_{h} y_{n}, y_{n}-w\right)_{\mathcal{H}}+\left(\Lambda_{h} w-\Lambda_{h} y_{n}, w-y\right)_{\mathcal{H}} \\
& \leq s\left(\Lambda_{h} w-\Lambda_{h} y_{n}, u-y\right)_{\mathcal{H}}
\end{aligned}
$$

we have

$$
\begin{aligned}
s\left(\Lambda_{h} y_{n}, y-u\right)_{\mathcal{H}} \geq & -\left(\Lambda_{h} y_{n}, y_{n}-y\right)_{\mathcal{H}}+\left(\Lambda_{h} w, y_{n}-y\right)_{\mathcal{H}} \\
& -s\left(\Lambda_{h} w, u-y\right)_{\mathcal{H}} \text { for all } n \geq 1,
\end{aligned}
$$

and

$$
s \lim _{n \rightarrow \infty}\left(\Lambda_{h} y_{n}, y-u\right)_{\mathcal{H}} \geq-s\left(\Lambda_{h} w, u-y\right)_{\mathcal{H}},
$$

so that $\underline{\lim }_{n \rightarrow \infty}\left(\Lambda_{h} y_{n}, y-u\right)_{\mathcal{H}} \geq-\left(\Lambda_{h} w, u-y\right)_{\mathcal{H}}$. and

Let $s \rightarrow 0^{+}$. Then $\underline{\lim }_{n \rightarrow \infty}\left(\Lambda_{h} y_{n}, y-u\right)_{\mathcal{H}} \geq-\left(\Lambda_{h} y, u-y\right)_{\mathcal{H}}=\left(\Lambda_{h} y, y-u\right)_{\mathcal{H}}$

$$
\begin{aligned}
\lim _{n \rightarrow \infty}\left(\Lambda_{h} y_{n}, y_{n}-u\right)_{\mathcal{H}} & \geq \lim _{n \rightarrow \infty}\left(\Lambda_{h} y_{n}, y_{n}-y\right)_{\mathcal{H}}+\lim _{n \rightarrow \infty}\left(\Lambda_{h} y_{n}, y-u\right)_{\mathcal{H}} \\
& \geq\left(\Lambda_{h} y, y-u\right)_{\mathcal{H}} \quad \text { for all } u \in \mathcal{H}
\end{aligned}
$$

Thus we have the required statement. Lemma 41 is proved.

Now Lemma 40 is also proved.

We continue now the proof of Theorem 39. We shall pass to the limit as $h \rightarrow 0^{+}$. From Lemma 40 for arbitrary $h>0$ the existence of $u_{h} \in \mathcal{H} \cap \mathcal{V}, d_{h}^{\prime} \in \mathcal{A}\left(u_{h}\right)$ and $d_{h}^{\prime \prime} \in \mathcal{B}\left(u_{h}\right)$ follows such that

$$
\Lambda_{h} u_{h}+d_{h}^{\prime}+d_{h}^{\prime \prime}=f
$$


and

$$
\left\|u_{h}\right\| \mathcal{V} \leq R \text { for any } h>0
$$

follows. From estimate (37) and the boundedness of the operator $\mathcal{A}$ on $\mathcal{V}_{1}$ it follows that

$$
\mathcal{A}\left(u_{h}\right) \text { is bounded in } \mathcal{V}_{1}^{\prime} \text { as } h \rightarrow 0 \text {. }
$$

Let us prove that

$$
d_{h}^{\prime \prime} \text { is bounded in } \mathcal{V}_{2}^{\prime} \text { as } h \rightarrow 0 \text {. }
$$

First, from (35), (36), estimate (37), the boundedness of the operator $\mathcal{A}$ and Proposition 6 we obtain that, for any $\left\{h_{n}\right\} \subset(0,+\infty)$ such that $h_{n} \rightarrow 0$, as $n \rightarrow \infty$,

$$
\begin{aligned}
\sup _{n}\left\langle d_{h_{n}}^{\prime \prime}, u_{h_{n}}\right\rangle \mathcal{V}_{2} & \leq \sup _{n}\left\langle f, u_{h_{n}}\right\rangle \mathcal{V}_{2}+\sup _{n}\left\langle-d_{h_{n}}^{\prime}, u_{h_{n}}\right\rangle \mathcal{V}_{2}+\sup _{n}\left\langle-\Lambda_{h_{n}} u_{h_{n}}, u_{h_{n}}\right\rangle \mathcal{V}_{2} \\
& \leq\|f\| \mathcal{V}^{\prime} \sup _{n}\left\|u_{h_{n}}\right\| \mathcal{V}+\sup _{n}\left\|u_{h_{n}}\right\| \mathcal{V} \sup _{n}\left\|\mathcal{A}\left(u_{h_{n}}\right)\right\|_{+}<+\infty
\end{aligned}
$$

Hence, owing to the quasi-boundedness of $\mathcal{B}$, estimate (39) follows.

From equality (36) and estimates (37)-(39), using the Banach-Alaoglu theorem, we obtain the existence of subsequences $\left\{u_{h_{h}}\right\}_{n \geq 1} \subset\left\{u_{h}\right\}_{h>0},\left\{d_{h_{n}}^{\prime}\right\}_{n \geq 1} \subset\left\{d_{h}^{\prime}\right\}_{h>0}$, $\left\{d_{h_{n}}^{\prime \prime}\right\}_{n \geq 1} \subset\left\{d_{h}^{\prime \prime}\right\}_{h>0}\left(0<h_{n} \rightarrow 0\right)$, denoted again by $\left\{u_{h}\right\}_{h>0},\left\{d_{h}^{\prime}\right\}_{h>0},\left\{d_{h}^{\prime \prime}\right\}_{h>0}$, and $u \in \mathcal{V}, d^{\prime} \in \mathcal{V}_{1}, d^{\prime \prime} \in \mathcal{V}_{2}$, such that

$$
u_{h} \rightarrow u \text { in } \mathcal{V}, \quad d_{h}^{\prime} \rightarrow d^{\prime} \text { in } \mathcal{V}_{1}^{\prime}, \quad d_{h}^{\prime \prime} \rightarrow d^{\prime \prime} \text { in } \mathcal{V}_{2}^{\prime}, \quad \Lambda_{h} u_{h} \rightarrow \Lambda u \text { in } \mathcal{V}^{\prime}
$$

From here, in particular, it follows that

$$
v_{h}:=d_{h}^{\prime}+d_{h}^{\prime \prime} \rightarrow d^{\prime}+d^{\prime \prime}:=w \text { in } \mathcal{V}^{\prime}
$$

Let us introduce the map $\mathcal{C}(v)=\mathcal{A}(v)+\mathcal{B}(v): \mathcal{V} \rightarrow C_{v}\left(\mathcal{V}^{\prime}\right)$. We shall prove that this map satisfies the property $(M)$ (see Definition 21). For this it is enough to show the $\lambda$-pseudomonotonicity of $\mathcal{C}$ on $\mathcal{V}$. Indeed, if $\mathcal{C}$ is $\lambda$-pseudomonotone on $\mathcal{V}$ and $\left\{y_{n}\right\}_{n \geq 0} \subset \mathcal{V}, d_{n} \in C\left(y_{n}\right)$, for all $n \geq 1$, are such that

$$
y_{n} \rightarrow y_{0} \text { in } \mathcal{V}, \quad d_{n} \rightarrow d_{0} \text { in } \mathcal{V}^{\prime} \quad \text { and } \varlimsup_{n \rightarrow \infty}\left\langle d_{n}, y_{n}\right\rangle_{\mathcal{V}} \leq\left\langle d_{0}, y_{0}\right\rangle_{\mathcal{V}}
$$

then

$$
\begin{aligned}
\overline{\lim }_{n \rightarrow \infty}\left\langle d_{n}, y_{n}-y_{0}\right\rangle_{\mathcal{V}} & \leq \overline{\lim _{n \rightarrow \infty}}\left\langle d_{n}, y_{n}\right\rangle_{\mathcal{V}}+\overline{\lim }_{n \rightarrow \infty}\left\langle d_{n},-y_{0}\right\rangle_{\mathcal{V}} \\
& \leq\left\langle d_{0}, y_{0}\right\rangle_{\mathcal{V}}-\left\langle d_{0}, y_{0}\right\rangle_{\mathcal{V}}=0
\end{aligned}
$$

Hence, thanks to the $\lambda$-pseudomonotonicity of $\mathcal{C}$ the existence of $\left\{y_{n_{k}}\right\}_{k \geq 1} \subset\left\{y_{n}\right\}_{n \geq 1}$, $\left\{d_{n_{k}}\right\}_{k \geq 1} \subset\left\{d_{n}\right\}_{n \geq 1}$ follows, such that

$$
\lim _{k \rightarrow \infty}\left\langle d_{n_{k}}, y_{n_{k}}-w\right\rangle_{\mathcal{V}} \geq\left[\mathcal{C}\left(y_{0}\right), y_{0}-w\right]_{-}=\left[\overline{\operatorname{co}}^{*} \mathcal{C}\left(y_{0}\right), y_{0}-w\right]_{-} \quad \text { for all } w \in \mathcal{V}
$$


From here

$$
\begin{aligned}
{\left[\mathcal{C}\left(y_{0}\right), y_{0}-w\right]_{-} } & \leq \lim _{k \rightarrow \infty}\left\langle d_{n_{k}}, y_{n_{k}}-w\right\rangle_{\mathcal{V}} \leq \varlimsup_{n \rightarrow \infty}\left\langle d_{n}, y_{n}-w\right\rangle_{\mathcal{V}} \\
& \leq\left\langle d_{0}, y_{0}-w\right\rangle_{\mathcal{V}} \quad \text { for all } w \in \mathcal{V} .
\end{aligned}
$$

Hence, Proposition 6 and $\mathcal{C}(y) \in C_{v}\left(\mathcal{V}^{\prime}\right)$ imply $d_{0} \in \mathcal{C}\left(y_{0}\right)$. Thus, $\mathcal{C}$ satisfies the property $(M)$ on $\mathcal{V}$.

Further, since $\mathcal{A}$ is a $\lambda$-pseudomonotone operator with bounded values on $\mathcal{V}_{1}$ and $\mathcal{B}$ is $\lambda$-pseudomonotone on $\mathcal{V}_{2}$, Lemma 33 implies that $\mathcal{C}$ is $\lambda$-pseudomonotone.

We use the fact that $\mathcal{C}$ satisfies the property $(M)$ on $\mathcal{V}$. Take $v$ from $\mathcal{V} \cap D\left(\Lambda^{*} ; \mathcal{V}^{\prime}\right)$. From (36) and (40) it follows that

$$
\left\langle u_{h}, \Lambda_{h}^{*} v\right\rangle_{\mathcal{V}}+\left\langle v_{h}, v\right\rangle_{\mathcal{V}}=\langle f, v\rangle_{\mathcal{V}}
$$

But

$$
\Lambda_{h}^{*} v=\frac{I-G(h)^{*}}{h} v+\frac{1-\theta_{h}}{h} G(h)^{*} v,
$$

and, by (30), $\Lambda_{h}^{*} v \rightarrow \Lambda^{*} v$ in $\mathcal{V}^{\prime}$. Consequently, passing to the limit in (41) as $h \rightarrow 0$ we shall obtain that

$$
\left\langle u, \Lambda^{*} v\right\rangle_{\mathcal{V}}+\langle w, v\rangle_{\mathcal{V}}=\langle f, v\rangle_{\mathcal{V}} \quad \text { for all } v \in \mathcal{V} \cap D\left(\Lambda^{*} ; \mathcal{V}^{\prime}\right)
$$

and then by (8) we get $u \in D\left(\Lambda, \mathcal{V}, \mathcal{V}^{\prime}\right)$ and

$$
\Lambda u+w=f .
$$

The proof of Theorem 39 will be finished if we can show that

$$
w \in \mathcal{C}(u)
$$

From (36) and (40) for $v \in \mathcal{V} \cap D\left(\Lambda ; \mathcal{V}^{\prime}\right) \subset \mathcal{H}$,

$$
\begin{aligned}
\left\langle v_{h}, u_{h}-v\right\rangle_{\mathcal{V}} & =\left\langle f, u_{h}-v\right\rangle_{\mathcal{V}}-\left\langle\Lambda_{h} v, u_{h}-v\right\rangle_{\mathcal{V}}-\left\langle\Lambda_{h}\left(u_{h}-v\right), u_{h}-v\right\rangle_{\mathcal{V}} \\
& \leq\left\langle f, u_{h}-v\right\rangle_{\mathcal{V}}-\left\langle\Lambda_{h} v, u_{h}-v\right\rangle_{\mathcal{V}},
\end{aligned}
$$

as $\Lambda_{h} \geq 0$ in $\mathcal{L}(\mathcal{H}, \mathcal{H})$. From here

$$
\begin{array}{r}
\lim \sup \left\langle v_{h}, u_{h}\right\rangle_{\mathcal{V}} \leq\langle w, v\rangle_{\mathcal{V}}+\langle f, u-v\rangle_{\mathcal{V}}-\langle\Lambda v, u-v\rangle_{\mathcal{V}} \\
\text { for all } v \in \mathcal{V} \cap D\left(\Lambda ; \mathcal{V}^{\prime}\right)
\end{array}
$$

But, by (10), the same inequality is fulfilled for any $v \in D\left(\Lambda ; \mathcal{V}, \mathcal{V}^{\prime}\right)$, and, putting $v=u$, we obtain

$$
\lim \sup \left\langle v_{h}, u_{h}\right\rangle_{\mathcal{V}} \leq\langle w, u\rangle_{\mathcal{V}}
$$

and (42) follows, as $\mathcal{C}$ is an operator of type $(M)$. Theorem 39 is proved. 
Now, Theorem 37 is a direct consequence of Theorem 39.

The next proposition follows from Definition 23 and Theorem 37.

PROPOSITION 42. Under the assumptions of Theorem 37 the problem

$$
u \in D\left(\Lambda ; \mathcal{V}, \mathcal{V}^{\prime}\right)
$$

$\langle\Lambda u, v-u\rangle_{\mathcal{V}}+[\mathcal{A}(u), v-u]_{+}+\varphi(v)-\varphi(u) \geq\langle f, v-u\rangle_{\mathcal{V}} \quad$ for all $v \in \mathcal{V}$

has a solution $u \in \mathcal{V}$. Furthermore, inequality (44) is equivalent to inclusion (4).

ProOF. Thanks to Proposition 6 it is enough to show that inequality (44) is equivalent to

$$
\langle\Lambda u, v-u\rangle_{\mathcal{V}}+[\mathcal{A}(u), v-u]_{+}+[\partial \varphi(u), v-u]_{+} \geq\langle f, v-u\rangle_{\mathcal{V}} \quad \text { for all } v \in \mathcal{V}
$$

This follows from Definition 23 and from the formula [1]

$$
D_{+} \varphi(u ; v-u):=\lim _{t \rightarrow 0^{+}} \frac{\varphi(u+t(v-u))-\varphi(u)}{t}=[\partial \varphi(u), v-u]_{+} .
$$

\section{Applications}

In this section we shall apply our main theorem to some particular equations.

EXAMPLE 1. Let $\Omega \subset \mathbb{R}^{n}$ be a bounded region with smooth boundary $\partial \Omega$, $S=[0, T]$ be a finite time interval, $Q=\Omega \times(0 ; T)$ and $\Gamma_{T}=\partial \Omega \times(0 ; T)$. The operator $\mathcal{A}$ is defined by $(\mathcal{A} u)(t)=\mathcal{A}(u(t))$, where

$$
\mathcal{A}(u)=-\sum_{i=1}^{n} \frac{\partial}{\partial x_{i}}\left(\left|\frac{\partial u}{\partial x_{i}}\right|^{p-2} \frac{\partial u}{\partial x_{i}}\right)+|u|^{p-2} u
$$

(see [10, Chapter 2.9.5]). Let $V$ be a closed subspace in the Sobolev space $W^{1, p}(\Omega)$, $p>1$, such that

$$
W_{0}^{1, p}(\Omega) \subset V \subset W^{1, p}(\Omega)
$$

We define the space

$$
H:=L^{2}(\Omega)
$$

and

$$
\mathcal{V}_{1}=L^{p}(0, T ; V), \quad \mathcal{H}=L^{2}(0, T ; H), \quad \mathcal{V}_{2}=L^{2}(0, T ; H)
$$

The operator $\mathcal{A}: \mathcal{V}_{1} \rightarrow \mathcal{V}_{1}^{\prime}$ is bounded, +-coercive and pseudomonotone (see [10, Chapter 2]). Thus, it is also $\lambda$-pseudomonotone. 
Let us consider a convex lower semicontinuous functional $\psi: \mathbb{R} \rightarrow \mathbb{R}$. Assume the existence of constants $M, C>0$ such that

$$
\psi(s) \geq M s^{2}+C \quad \text { for all } u,
$$

and also that $\psi(u) \in L^{1}((0, T) \times \Omega)$, for all $u \in \mathcal{H}$. Denote by $\Phi: \mathbb{R} \rightrightarrows \mathbb{R}$ its subdifferential. It is well known [2, p. 61] that $\varphi: \mathcal{V}_{2} \rightarrow \mathbb{R}$ defined by

$$
\varphi(u)=\int_{0}^{T} \int_{\Omega} \psi(u(x)) d x,
$$

is a convex, lower semicontinuous function in $\mathcal{V}_{2}$. Moreover, $w \in \partial \varphi(u)$ if and only if $w(x) \in \Phi(u(x))$, almost everywhere on $(0, T) \times \Omega$, and $w_{i} \in \mathcal{V}_{2}$. It follows easily from (47) that $\mathcal{B}=\partial \varphi$ is + -coercive.

Putting $\mathcal{V}=\mathcal{V}_{1} \cap \mathcal{V}_{2}$ (and then $\mathcal{V}^{\prime}=L^{q}\left(0, T ; V^{\prime}\right)+L^{2}\left(0, T ; L^{2}(\Omega)\right)$ ), where $(1 / p)+(1 / q)=1$, we have that (6) holds if $p \geq 2$. For $1<p<2$ we can take $\Phi=\mathcal{D}(0, T ; V)$, the space of infinitely continuously differentiable functions with compact support (see [10]).

In our case $\Lambda=d y / d t$ is the derivative in the sense of scalar distributions $\mathcal{D}^{\prime}\left(0, T ; V^{\prime}\right)$ and

$$
\begin{gathered}
D\left(\Lambda ; \mathcal{V}, \mathcal{V}^{\prime}\right):=W=\left\{y \in \mathcal{V} \cap \mathcal{H}: y^{\prime} \in \mathcal{H}+\mathcal{V}^{\prime}, y(0)=\overline{0}\right\}, \\
G(s) u(t):= \begin{cases}u(t-s) & \text { for } t \geq s, \\
0 & \text { for } t \leq s .\end{cases}
\end{gathered}
$$

The map $\Lambda$ satisfies conditions (7)-(10) [10, Section 2.9].

Then all the conditions of Theorem 37 are satisfied, so that the problem

$$
\begin{aligned}
\int_{Q} & \frac{d y(x, t)}{d t}(v(x, t)-y(x, t)) d x d t+\sum_{i=1}^{n} \int_{Q}\left|\frac{\partial y(x, t)}{\partial x_{i}}\right|^{p-2} \frac{\partial y(x, t)}{\partial x_{i}} \\
& \times\left(\frac{\partial v(x, t)}{\partial x_{i}}-\frac{\partial y(x, t)}{\partial x_{i}}\right) d x d t+\int_{Q}|y|^{p-2} y(v-y) d x d t \\
& +\int_{Q} \psi(v(x, t)) d x d t-\int_{Q} \psi(y(x, t)) d x d t \\
\geq & \int_{Q} f(v-y) d x d t+\int_{\Gamma_{T}} g(v-y) d x d t \quad \text { for all } v \in \mathcal{V}, \\
y(x, 0)= & 0 \quad \text { almost everywhere on } \Omega,
\end{aligned}
$$

has a solution $y \in W$, obtained by the method of difference approximations. Note that, in (48)-(49), $f \in L^{2}(Q), g \in L^{2}\left(\Gamma_{T}\right)$ are fixed elements.

EXAMPLE 2 . Let $n \geq 1, k \geq 1, A \subset \mathbb{R}^{k}$ be a non-empty compact set and $\Omega \subset \mathbb{R}^{n}$ be a bounded region with smooth boundary $\partial \Omega$. Let us also consider a family of maps $U_{\alpha}: \mathbb{R}^{n} \rightarrow \mathbb{R}$, where $\alpha \in A$, that satisfies the following conditions: 
(1) the $\operatorname{map} \mathbb{R}^{n} \times A \ni(\xi, \alpha) \rightarrow U_{\alpha}(\xi) \in \mathbb{R}$ is continuous;

(2) $\mathbb{R}^{n} \ni \xi \rightarrow U_{\alpha}(\xi) \in \mathbb{R}$ is convex for all $\alpha \in A$;

(3) there exist $a>0, \quad b>0$ such that $\left\|\partial U_{\alpha}(\xi)\right\|_{+} \leq a+b\|\xi\|$ for all $\xi \in \mathbb{R}^{n}$, for all $\alpha \in A$.

Together with $\left\{U_{\alpha}(\xi)\right\}_{\alpha \in A}$ let us consider the function $U(\xi):=\max _{\alpha \in A} U_{\alpha}(\xi)$ : $\mathbb{R}^{n} \rightarrow \mathbb{R}$ and the multi-valued map with compact values $G(\xi):=\left\{\alpha \in A \mid U_{\alpha}(\xi)=\right.$ $U(\xi)\}, \xi \in \mathbb{R}^{n}$. Assume also the following coercitivity condition:

(4) there exist constants $M, C>0$ such that

$$
U(\xi) \geq M\|\xi\|^{2}+C \text { for all } \xi .
$$

Let us consider the following problem:

$$
\begin{gathered}
y^{\prime}(t, x)-\sum_{i=1}^{n} \frac{\partial}{\partial x_{i}}\left(\left|\frac{\partial y(t, x)}{\partial x_{i}}\right|^{p-2} \frac{\partial y(t, x)}{\partial x_{i}}\right) \\
-\sum_{i=1}^{n} \frac{\partial}{\partial x_{i}} \overline{c o}\left(\bigcup_{\substack{\left.\alpha \in G(\nabla y(t, x)) \\
y(t, x)\right|_{\partial \Omega}=0, y(0, x)=0 .}} \partial U_{\alpha}(\nabla y(t, x))\right) \ni f(t, x),
\end{gathered}
$$

From [16, Theorem II.3.14] and the given conditions it follows that inclusion (50) is equivalent to

$$
\begin{aligned}
& y^{\prime}(t, x)-\sum_{i=1}^{n} \frac{\partial}{\partial x_{i}}\left(\left|\frac{\partial y(t, x)}{\partial x_{i}}\right|^{p-2} \frac{\partial y(t, x)}{\partial x_{i}}\right) \\
& -\sum_{i=1}^{n} \frac{\partial}{\partial x_{i}} \partial U(\nabla y(t, x)) \ni f(t, x) .
\end{aligned}
$$

Then we obtain the following differential-operator inclusion

$$
y^{\prime}+\mathcal{A}(y)+L^{*} \partial \varphi(L y) \ni f, \quad y(0)=\overline{0},
$$

where

$$
\begin{gathered}
\mathcal{A}: L^{p}\left(0, T ; H_{0}^{1}(\Omega)\right) \rightarrow L^{q}\left(0, T ; H^{-1}(\Omega)\right), \\
L: H_{0}^{1}(\Omega) \rightarrow\left(L^{2}(\Omega)\right)^{n}\left(L v=\nabla v, \text { for all } v \in H_{0}^{1}(\Omega)\right), \\
L^{*}:\left(L^{2}(\Omega)\right)^{n} \rightarrow H^{-1}(\Omega)\left(L^{*} v=-\operatorname{div} v \text {, for all } v \in L^{2}(\Omega)\right), \\
\varphi: L^{2}\left(0, T ;\left(L^{2}(\Omega)\right)^{n}\right) \rightarrow \mathbb{R}, \\
\varphi(z)=\int_{Q} U(z(t, x)) d t d x \quad \text { for all } z \in L^{2}\left(0, T ;\left(L^{2}(\Omega)\right)^{n}\right), \\
f \in L^{2}\left(0, T ; L^{2}(\Omega)\right)+L^{q}\left(0, T ; H^{-1}(\Omega)\right),
\end{gathered}
$$

and $\mathcal{V}_{1}=L^{p}\left(0, T ; H_{0}^{1}(\Omega)\right), \mathcal{H}=L^{2}\left(0, T ; L^{2}(\Omega)\right), \mathcal{V}_{2}=L^{2}\left(0, T ; H_{0}^{1}(\Omega)\right)$. 
Hence, inclusion (54) is equivalent to

$$
y^{\prime}+\mathcal{A}(y)+\partial(\varphi \circ L)(y) \ni f, \quad y(0)=\overline{0},
$$

(see [6]). It is easy to see that $\varphi \circ L: \mathcal{V}_{2} \rightarrow \mathbb{R}$ is convex, lower semicontinuous and coercive. So, in a similar way as in the previous example, from conditions (1)-(4) we obtain that problem (50)-(52) has a solution $y \in L^{2}\left(0, T ; H_{0}^{1}(\Omega)\right)$ $\cap L^{p}\left(0, T ; H_{0}^{1}(\Omega)\right)$.

\section{References}

[1] J. P. Aubin and I. Ekeland, Applied nonlinear analysis (Mir, Moscow, 1988).

[2] V. Barbu, Nonlinear semigroups and differential equations in Banach spaces (Editura Acad., Bucuresti, 1976).

[3] H. Brezis, 'Problems unilatéraux', J. Math. Pures Appl. 51 (1972), 1-168.

[4] F. E. Browder and P. Hess, 'Nonlinear mappings of monotone type in Banach spaces', J. Funct. Anal. 11 (1972), 251-294.

[5] Yu. A. Dubinski, 'Weak convergence in non-linear elliptic and parabolic equations', Mat. Sb. 67 (1965), 609-642 (translated in Am. Math. Soc., II, Ser. 67, (1968), 226-258).

[6] I. R. Ekeland and R. Temam, Convex analysis and variational problems (North-Holland, Amsterdam, 1976).

[7] P. O. Kasyanov, 'Galerkin method for a class of differential-operator inclusions with set-valued mappings of pseudomonotone type', Naukovi Visti NTUU 'KPI' 2 (2005), 139-151.

[8] _ 'Galerkin's method for one class of differential-operator inclusions', Dopov. Nats. Akad. Nauk Ukr. 9 (2005), 20-24.

[9] P. O. Kasyanov and V. S. Melnik, 'Faedo-Galerkin method differential-operator inclusions in Banach spaces with maps of $w_{\lambda_{0}}$-pseudomonotone type', Nats. Acad. Sci. Ukr., Kiev, Inst. Math., Prepr. 1(Part 2) (2005), 82-105.

[10] J. L. Lions, Quelques methodes de resolution des problemes aux limites non lineaires (Dunod Gauthier-Villars, Paris, 1969).

[11] V. S. Melnik, 'Multivariational inequalities and operational inclusions in Banach spaces with maps of a class $(S)_{+}$', Ukr. Mat. Zh. 52 (2000), 1513-1523.

[12] _ _ 'About critical points of some classes multivalued maps', Cybernet. Systems Anal. 2 (1997), 87-98.

[13] _ 'About operational inclusions in Banach spaces with densely defined operators', Syst. Res. Inform. Technol. 3 (2003), 120-126.

[14] V. S. Melnik and M. Z. Zgurovsky, Nonlinear analysis and control of physical processes and fields (Springer, Berlin, 2004).

[15] - 'Ky Fan inequality and operational inclusions in Banach spaces', Cybernet. Systems Anal. 2 (2002), 70-85.

[16] B. Pshenichniy, Convex analysis and extremal problems (Nauka, Moscow, 1980).

[17] M. Reed and B. Simon, Methods of mathematical physics 1: Functional analysis (Academic Press, New York, 1980).

[18] W. Rudin, Functional analysis (McGraw-Hill, New York, 1973).

[19] I. V. Skripnik, Methods of investigation of nonlinear elliptic boundary problems (Nauka, Moscow, 1990).

[20] A. A. Tolstonogov, 'About solutions of evolutionary inclusions 1', Siberian Math. J. 33 (1992), $145-162$. 
[21] A. A. Tolstonogov and J. I. Umanski, 'About solutions of evolutionary inclusions 2', Siberian Math. J. 33 (1992), 163-174.

[22] M. M. Vainberg, Variational methods and method of monotone operators (Wiley, New York, 1973).

[23] A. N. Vakulenko and V. S. Melnik, 'Solvability and properties of solutions of one class of operator inclusions in Banach spaces', Naukovi Visti NTUU 'KPI' 3 (1999), 105-112.

[24] _ ' 'On a class of operator inclusions in Banach spaces', Dopov. Nats. Akad. Nauk Ukr. 8 (1998), 20-25.

[25] _ ' 'On topological method in operator inclusions with densely defined mappings in Banach spaces', Nonlinear Boundary Value Probl. 10 (2000), 125-142.

Kyiv Taras Shevchenko University

$01033 \mathrm{Kiev}$

Ukraine

e-mail: kasyanov@univ.kiev.ua

Centro de Investigación Operativa

Universidad Miguel Hernández

Avda Universidad s/n

03202 Elche, Alicante

Spain

e-mail: jvalero@umh.es
Institute of Applied and System Analysis

Kiev

Ukraine

e-mail: moreva@mmsa.ntu-kpi.kiev.ua 